ISSN 0280-5316 ISRN LUTFD2/TFRT--5687--SE

\title{
Modelling of Microturbine Systems
}

Staffan Haugwitz

Department of Automatic Control

Lund Institute of Technology

May 2002 



\section{Department of Automatic Control Lund Institute of Technology Box 118 SE-221 00 Lund Sweden}

Author(s)

Staffan Haugwitz

Document name

MASTER THESIS

Date of issue

May 2002

Document Number

ISRN LUTFD2/TFR--5687--SE

Supervisor

Anders Rantzer and Hubertus Tummescheit (LTH)

Göran Stångberg (Turbec AB)

Sponsoring organization

Title and subtitle

Modelling of Microturbine Systems (Modellering av mikroturbinsystem)

Abstract

The thesis describes the development of a dynamic model of a microturbine system. The thesis is done in close cooperation with Turbec AB and the model is adjusted, tuned and verified against their microturbine T100. The microturbine unit consists of a compressor and a turbine connected on a single shaft to a high-speed generator. Moreover there is a combustion chamber, a recuperator and a gas/water heat exchanger. A control system regulates the speed, the temperature and the electric power. To control the frequency, voltage and current of the outgoing power, the microturbine uses power electronics. The model is to be used in the research and development department at Turbec AB. Possible applications are in areas as control strategies, dynamic performance verification, operator training and control software/hardware verification. Since the potential applications are so different, the emphasis throughout the thesis has been on a general model that can be used in as many different operating ranges as possible. This should be done while retaining a high degree of accuracy at the most common scenario, running on full load. The emphasis has also been on the functionality and accuracy of the complete model over more detailed modelling of each component.

In the thesis, the thermodynamic theory of each component is described and how it is modelled in Modelica. The microturbine model in the thesis covers the gas turbine unit, the control system and the mechanical part of the generator. The electric part of the generator and the power electronics are not included in the model, due to the limited time of the project. The thesis also discusses the well-known problems with modelling as e.g. discretisation and interpolation. The model uses components from the ThermoFluid library, the Modelica standard library and the Master's thesis of Perez (2001).

Static verification has been done with data from DSA, a steady state calculation program, which accurately represents the real microturbine. The Modelica model developed in the thesis has been found very accurate at the main operation range $(100 \mathrm{~kW}$ down to $50 \mathrm{~kW})$, with an average error of $0.6 \%$ of the 13 most important thermodynamic variables at full load, $100 \mathrm{~kW}$. Dynamic verification in three different scenarios has been done against the real microturbine and the model shows a good fit to the measured data.

Keywords

Classification system and/or index terms (if any)

Supplementary bibliographical information

\begin{tabular}{|c|c|c|}
\hline $\begin{array}{l}\text { ISSN and } \\
0280-53\end{array}$ & & \\
\hline $\begin{array}{l}\text { Language } \\
\text { English }\end{array}$ & $\begin{array}{l}\text { Number of pages } \\
55\end{array}$ & Recipient's notes \\
\hline
\end{tabular}

The report may be ordered from the Department of Automatic Control or borrowed through:

University Library 2, Box 3, SE-221 00 Lund, Sweden

Fax $+46462224422 \quad$ E-mail ub2@ub2.se 



\section{Modelling of \\ Microturbine Systems}

Staffan Haugwitz

Master's Thesis

Lund Institute of Technology

May 2002

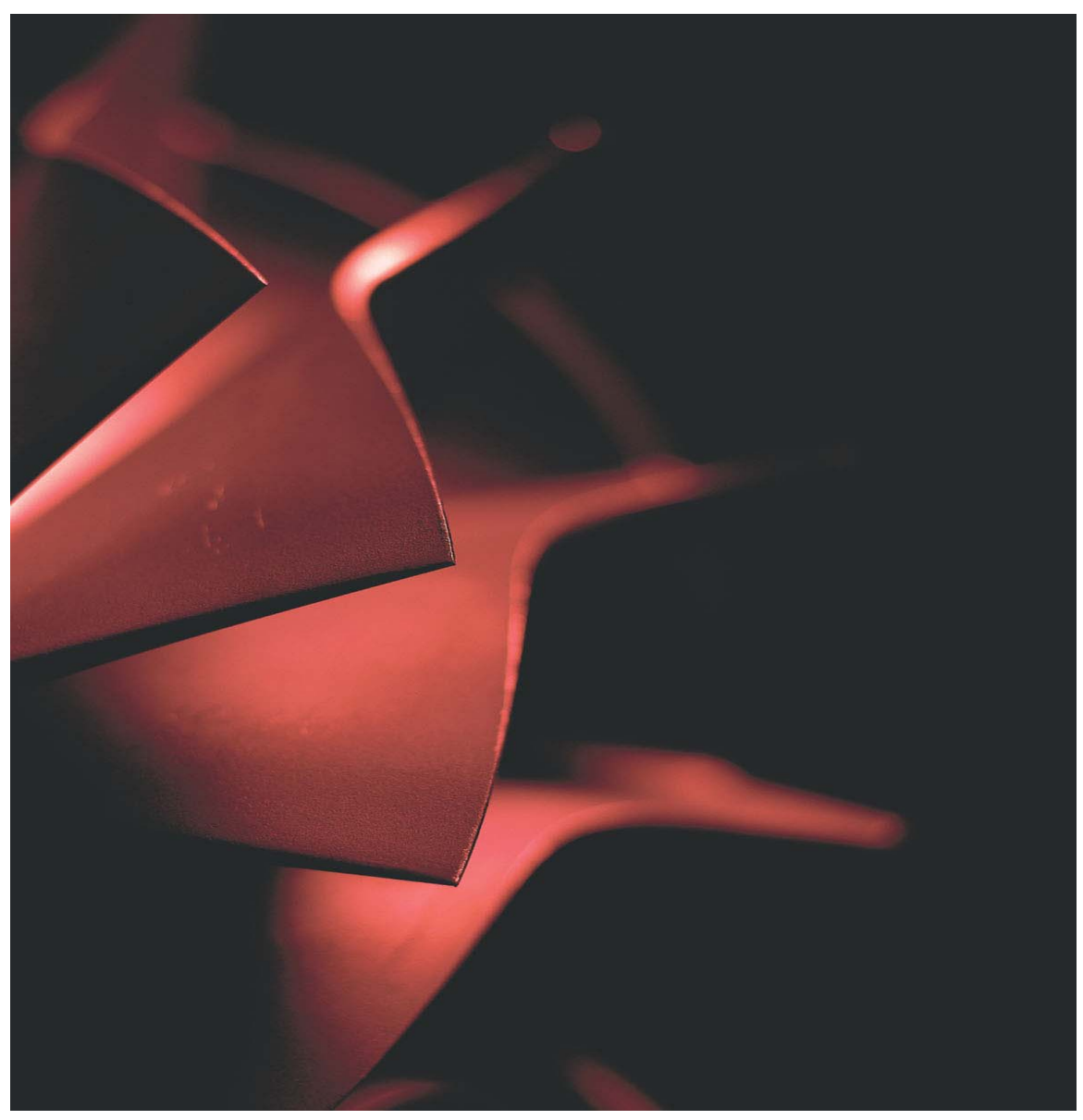




\section{Table of Content}

PREFACE .................................................................................................................................................. 5

NOMENCLATURE ............................................................................................................................................. 6

1. INTRODUCTION ................................................................................................................................... 7

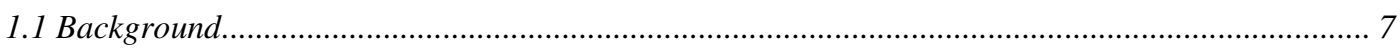

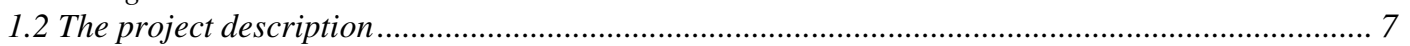

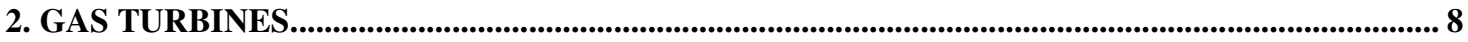

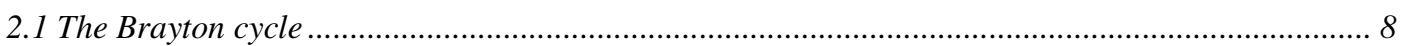

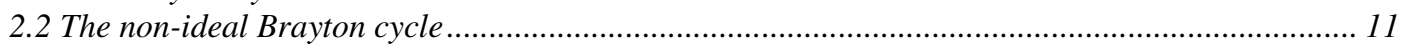

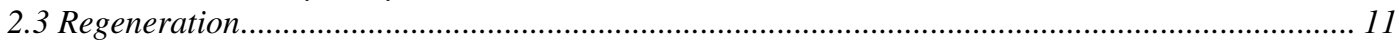

3. THE T100 MICROTURBINE SYSTEM.................................................................................. 12

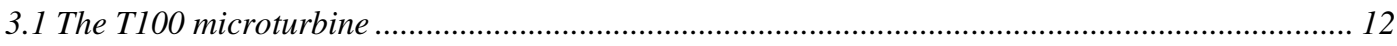

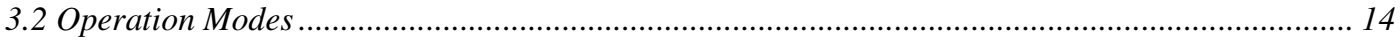

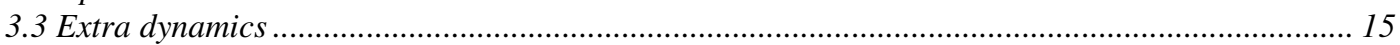

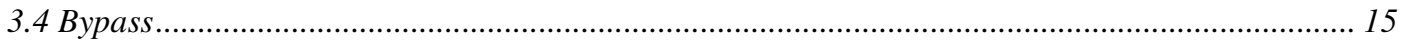

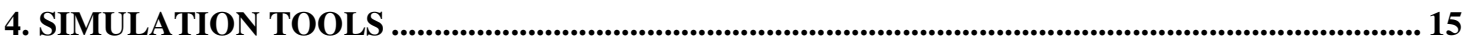

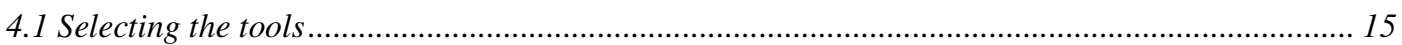

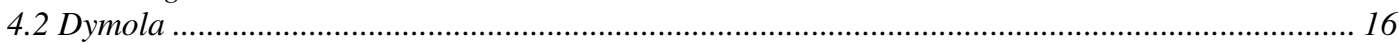

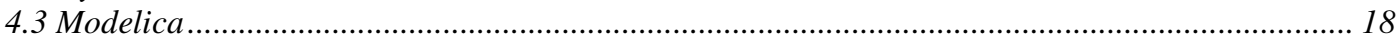

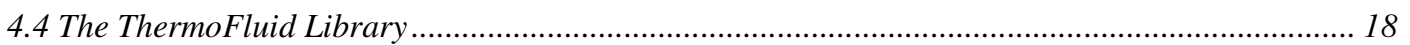

5. THERMODYNAMIC THEORY AND MODELLING ...................................................................... 22

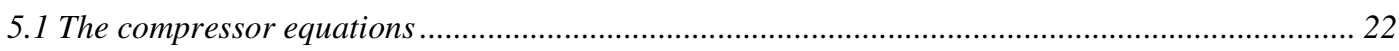

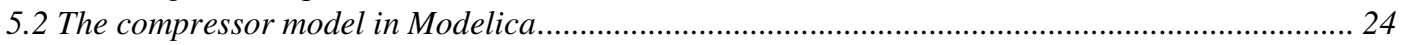

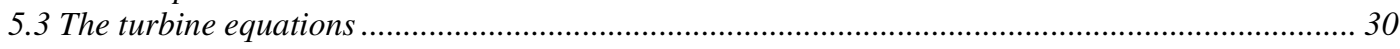

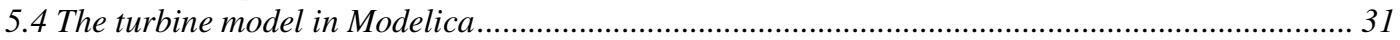

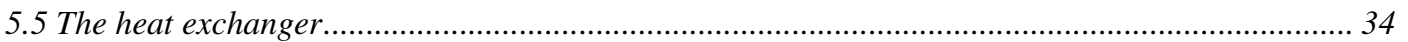

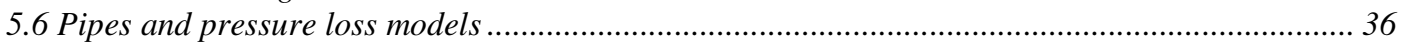

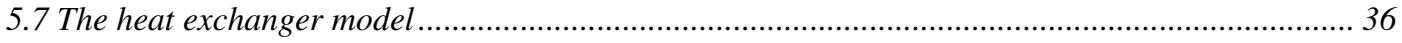

5.8 The combustion chamber ............................................................................................... 38

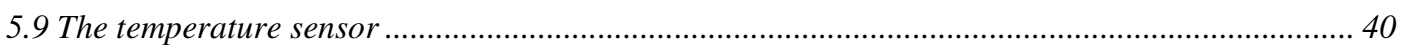

5.10 The Generator, Friction losses and Auxiliary equipment models...........................................41

5.11 Flow Sources and Reservoirs .............................................................................. 41

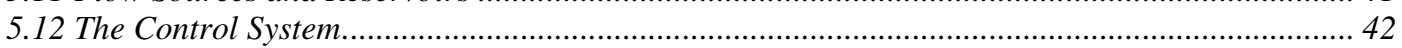

6. SIMULATIONS AND VERIFICATION ............................................................................................... 42

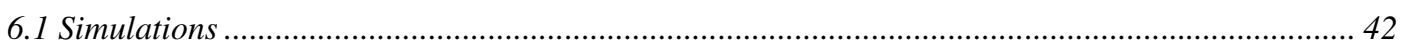

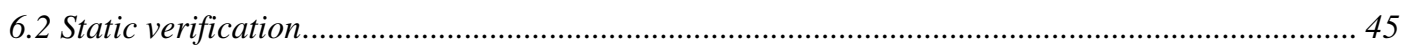

6.3 Dynamic verification …………………………………………………………………. 47

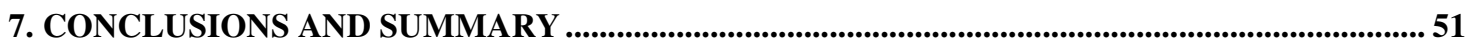

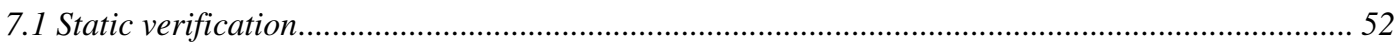

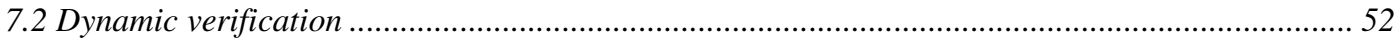

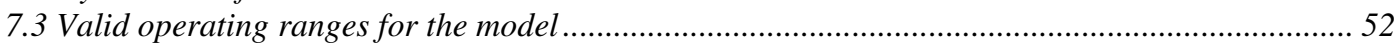

8. POSSIBLE APPLICATIONS AND FUTURE WORK......................................................................... 52

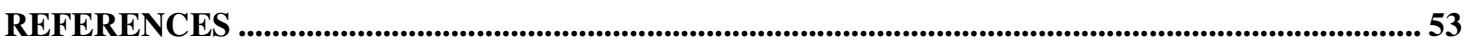




\section{Preface}

This thesis is concerned with the development of a thermodynamic simulation model of a microturbine. It was written at Turbec $\mathrm{AB}$ in cooperation with the institution of Automatic Control at Lund Institute of Technology. Turbec AB is a newly founded company (1998), jointly owned by Volvo Aero AB and ABB. In 1992 Volvo presented the Environmental Concept Car (ECC), which had a turbine-generator system based on a high-speed generator directly coupled with a simple single-shaft gas turbine. The gas turbine constantly charged batteries, which powered the vehicle.

The knowledge and experience from the mobile microturbines led to the development of a new technology, stationary combined heat and power (CHP) plants on a miniature scale. Volvo brought in $\mathrm{ABB}$ as a partner and formed the independent company Turbec $\mathrm{AB}$. The first product of the company is the T100, a microturbine that can produce $100 \mathrm{~kW}$ of electric power and another $167 \mathrm{~kW}$ of heat with a total efficiency of $80 \%$. The market of microturbines did not exist until 5 years ago, when Honeywell presented their first product. Now the market is growing rapidly. Due to confidentiality, some of the figures in this thesis are presented rescaled and without axes.

I would like to thank my tutors Anders Åberg (Turbec AB), Hubertus Tummescheit (LTH) and Anders Rantzer (LTH) for their help and enthusiasm throughout my work with the thesis.

Lund, May 2002

Staffan Haugwitz 


\section{Nomenclature}

In the thesis numerous variables are used and sometimes the same symbol is used for different meanings. Given below is a short list of used variables and symbols. Different text sources use different symbols for the same physical variable, e.g. in DeWitt (1996) the convection heat transfer coefficient is denoted by $h$, but in Gustafsson (1998) it is denoted by $\alpha$. I have in the thesis chosen the latter, to avoid confusion with specific enthalpy.

\begin{tabular}{|c|c|c|}
\hline Symbol & Physical Meaning & Unit \\
\hline$A$ & surface area & $\mathrm{m}^{2}$ \\
\hline$\alpha$ & convection heat transfer coefficient & $\mathrm{W} /\left(\mathrm{m}^{2} \mathrm{~K}\right)$ \\
\hline$c_{p}$ & specific heat capacity at constant pressure & $\mathrm{J} /(\mathrm{kg} \mathrm{K})$ \\
\hline$c_{v}$ & specific heat capacity at constant volume & $\mathrm{J} /(\mathrm{kg} \mathrm{K})$ \\
\hline$C$ & heat capacity flow & $\mathrm{J} /(\mathrm{K} \mathrm{s})$ \\
\hline$D$ & diameter & $\mathrm{m}$ \\
\hline$\varepsilon$ & efficiency/effectiveness & dimensionless \\
\hline$h$ & specific enthalpy & $\mathrm{J} / \mathrm{kg}$ \\
\hline$\kappa$ & ratio of specific heats & dimensionless \\
\hline$L$ & length & $\mathrm{m}$ \\
\hline$k$ & thermal conductivity & $\mathrm{W} /(\mathrm{m} \mathrm{K})$ \\
\hline$\dot{m}$ & mass flow rate & $\mathrm{kg} / \mathrm{s}$ \\
\hline$\dot{m}_{\text {corr }}$ & corrected mass flow rate & dimensionless \\
\hline$m$ & mass & $\mathrm{kg}$ \\
\hline$M$ & molar mass & $\mathrm{kg} / \mathrm{kmol}$ \\
\hline$n$ & rotational speed & $\mathrm{rpm}$ \\
\hline$n_{\text {corr }}$ & corrected rotational speed & dimensionless \\
\hline$\eta$ & efficiency & dimensionless \\
\hline$N T U$ & number of thermal units & dimensionless \\
\hline$p$ & pressure & $\mathrm{Pa}$ \\
\hline$P$ & power & $\mathrm{W}$ \\
\hline$\rho$ & density & $\mathrm{kg} / \mathrm{m}^{3}$ \\
\hline$q$ & specific heat flow & $\mathrm{J} / \mathrm{kg}$ \\
\hline$R$ & universal gas constant & $\mathrm{J} /(\mathrm{kg} \mathrm{K})$ \\
\hline$s$ & specific entropy & $\mathrm{J} /(\mathrm{kg} \mathrm{K})$ \\
\hline$t$ & time & $\mathrm{s}$ \\
\hline$\tau$ & torque & $\mathrm{Nm}$ \\
\hline$T$ & temperature & $\mathrm{K}$ \\
\hline$u$ & specific internal energy & $\mathrm{J} / \mathrm{kg}$ \\
\hline$U$ & overall heat transfer coefficient & $\mathrm{W} /\left(\mathrm{m}^{2} \mathrm{~K}\right)$ \\
\hline$v$ & specific volume & $\mathrm{m}^{3} / \mathrm{kg}$ \\
\hline$V$ & volume & $\mathrm{m}^{3}$ \\
\hline$w$ & specific work & $\mathrm{J} / \mathrm{kg}$ \\
\hline$\omega$ & angular velocity & $\mathrm{rad} / \mathrm{s}$ \\
\hline
\end{tabular}




\section{Introduction}

\subsection{Background}

In this section, I will briefly explain the market idea behind the microturbine systems. How come that these small units can compete with larger power plants?

In recent years a slow deregulation of the power generation market has taken place throughout the world. It is open to anyone to produce its own electricity and to export it to others. The market is also open to international corporations and monopolies in many countries are breaking up. The power corporations that produce energy in large complex facilities are now exposed to competition from small-scale production of power on site where the need is. State-owned power companies that were controlled by the government are now sold out on the stock market. The new owners tend to have a shorter perspective and value short- or medium-time profits. Long-term commitments are less frequent. The private companies do not want to maintain a large production buffer to assure that enough power is produced in case of extreme weather or other crisis. The power demand changes quickly in size and location. The prize on power changes also rapidly world wide due to speculations, politics and natural disasters.

All these changes make it harder for companies to justify the investments that a new full-scale power plant requires. An interesting example is California, USA where almost no new power plants have been built during the 8 years since the power market was deregulated in 1996; a situation which made the power shortages during January in 2001 more severe.

This development has lead to a new interest in small-scale power plants that are easy and quick to install, with low cost and a short payback time. The power production can be adjusted to the current demand and if the demand increases over time, another turbine can be installed. The companies do not need to take the full investment that a larger power plant would take. Smallscale power production can be viewed with the motto: invest as you grow.

Small-scale power plants are nothing new. But now it can be combined with water heating, thus increasing the total efficiency from 30 to $80 \%$ ! The combination of power and heat (CHP) generation is therefore essential to the success of the microturbine system.

The development of small-size on-site power plants increases also the flexibility in the power generation, e.g. when they are placed in clusters and connected into networks to serve many customers at many different locations. These distributed micro-grids can e.g. be operated as centralized systems monitored from a single location.

\subsection{The project description}

The microturbine T100 CHP is now being sold all over the world, with new versions and applications coming up. In order to verify new control strategies, e.g. how to control four microturbines connected in parallel, it is essential to have a reliable model to evaluate the static and dynamic effects. When designing new braking strategies for over speed protection it is also essential to have a model to work with. With a simulation model they can try these ideas out in a safe and timesaving way, instead of using a real machine. The purpose of this thesis is to give the engineers this simulation model.

As will be explained in chapter on Simulation Tools, no existing model at Turbec covers all aspects. The objective with the thesis is therefore to develop a thermodynamic simulation model 
for later use in various applications, e.g. the software development of the control system. Other applications might be to test hardware components from the control system connected to the real $\mathrm{I} / \mathrm{O}$ interface, but with a PC doing hardware in the loop simulation. The model can also be used as a tool in operator training, i.e. how to operate the machine with its user interface but with a simulation model instead of the real microturbine.

After some discussions (see the chapter on Simulation Tools) a decision was made to write the model in the language Modelica and use the simulation program Dymola from Dynasim AB, Lund. The model should also be extendable to cover the power electronics in a future work. It should be possible to export the model to other simulation environments as e.g. Matlab/Simulink. The model is to be verified against other simulation programs and real data from experiments. The model should be as complete as possible, including extra components, e.g. the recuperator and gas/water heat exchanger.

This thesis is a continuation of the Master's thesis by Perez (2001), where the basic structure of gas turbine modelling was outlined. The model structure of some of the components, e.g. the compressor and the turbine, was reused in the new model. Even though the machine modelled in that thesis was physically larger and produced $600 \mathrm{~kW}$ of power, the thermodynamic equations are the same. All the parameters, e.g. the ones containing the characteristics of e.g. compressor and turbine, had to be changed. The reuse of Perez's models saved large amounts of time and more efforts could therefore be made in parameter tuning and verification of the model, something that was beyond the scope of his thesis. Still one must emphasize that tuning a dynamic model with hundreds of parameters is a very delicate matter and the most important objective is to achieve a sound and reliable dynamic model with a thorough physical background.

In the chapters about Modelica, ThermoFluid and the derivation of the thermodynamic equations I have a similar approach to the one used in Perez (2001) since our base sources e.g. Cohen (1998) and Cengel (1999) are the same. I want to avoid repeating the same texts and equations as in Perez (2001), but in order to make my thesis complete and understandable to read without having the references, I have decided to include similar text and derivations.

\section{Gas Turbines}

In this chapter I will describe the general features of a gas turbine, the governing equations and the different steps in the ideal and the real Brayton cycle.

\subsection{The Brayton cycle}

The figure below shows the ideal open Brayton cycle. Fresh air is drawn into to compressor. 


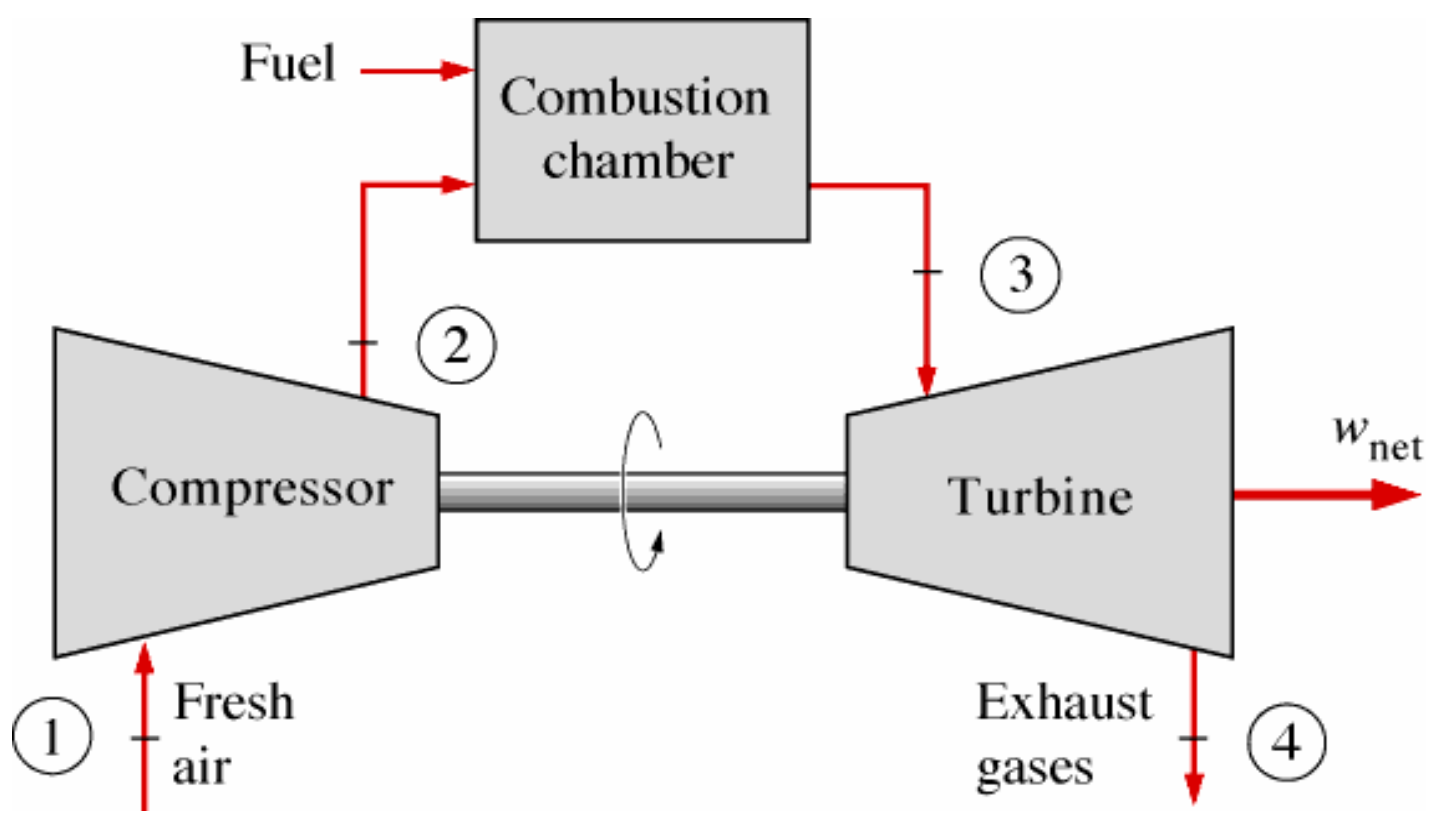

Figure 1: An open ideal Brayton cycle, Cengel (1998)

The compressor at stage 1 increases the pressure of the fresh air with a factor $4-20$ depending on its size and construction. At stage 2 the high-pressure air and fuel are mixed and burnt in the combustion chamber at a constant pressure. The very hot flue gas enters the turbine at stage 3 and forces the turbine to rotate, thus producing mechanical work. During this procedure the flue gas expands to lower pressure and larger volume, therefore the turbine is also called an expander. At stage 4 the exhaust gas is released to the surroundings. Since there are mass flows in and out of the process, it is called an open cycle. It can be remodelled as a closed cycle, i.e. there is no mass flow in or out of the process, by doing two approximations in the process. Firstly, the combustion chamber is replaced by a heat exchanger where the air is heated to the same temperature as it would get during combustion, but now no fuel is added. Secondly, another heat exchanger is placed between stage 4 and 1 such that the exhaust gas is cooled by the surroundings to the temperature at stage 1 . Then the same fluid is used throughout the process, hence the name closed cycle. The closed cycle is often used for thermodynamic analysis. 


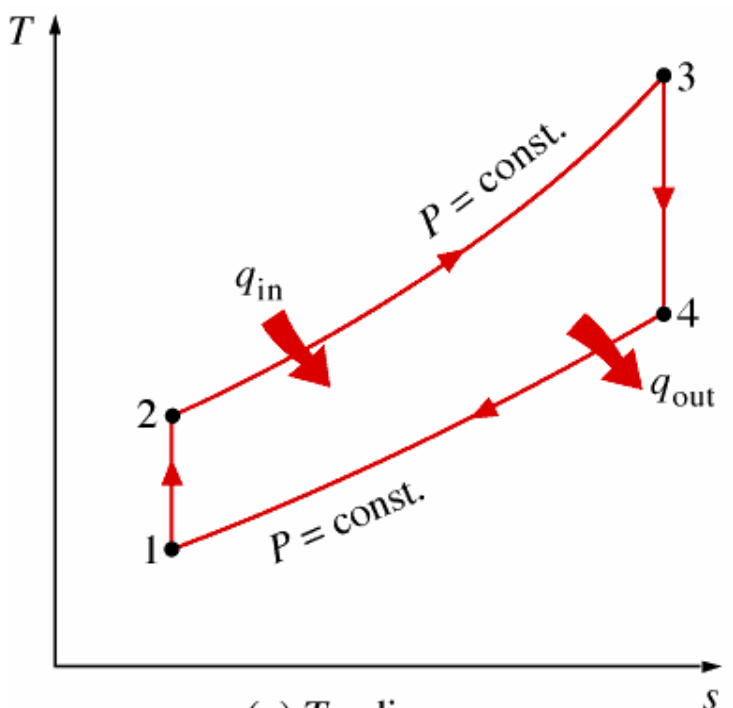

(a) $T$-s diagram

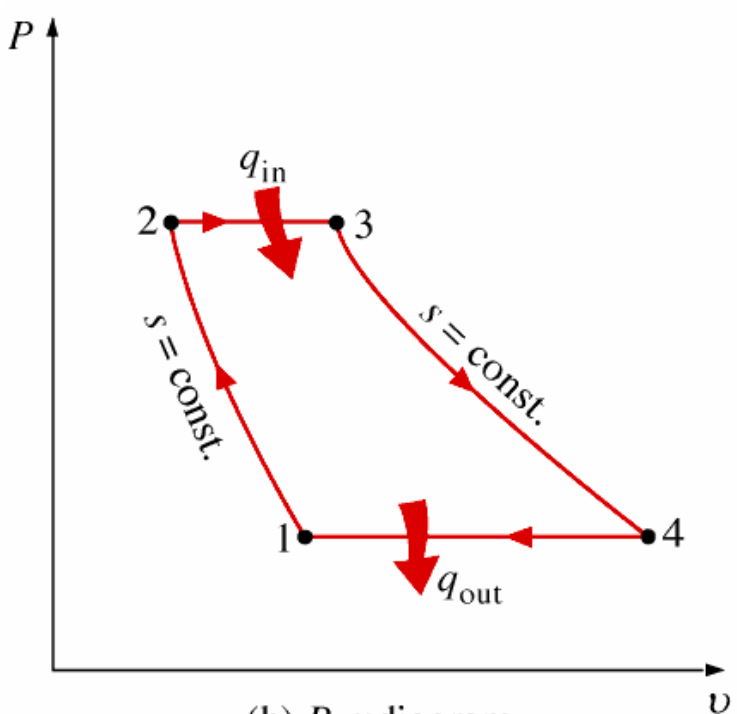

(b) $P$-vdiagram

Figure 2: T-s and p- $v$ diagrams for the ideal closed Brayton cycle, Cengel (1998)

In the figure to the right above, we can see how the pressure and specific volume change throughout the cycle and in the figure to the left above, the change in temperature and entropy is shown. Specific volume is defined as the inverse of the density, i.e. how much volume a unit mass occupies. During compression, the pressure and temperature increase. The pressure increase tends to increase the density, whereas the increase in temperature tends to decrease the density. The total effect is a small increase in density, which corresponds to a small decrease in specific volume. In an ideal cycle the compression is isentropic, i.e. adiabatic (no heat loss) and internally reversible (the entropy does not change due to friction etc). Therefore the entropy is constant even though the temperature increases.

In the combustion chamber heat is added to the gas at constant pressure. The density decreases and the specific volume and temperature are increased. Entropy is also increased, since combustion is not a reversible process. In the turbine the situation is the opposite of the compressor; the pressure decreases and specific volume increases. The temperature decreases and in an ideal expansion the entropy is constant.

From basic thermodynamics we know that the encircled area in the p- $v$ diagram represents the produced net work of the gas turbine. The efficiency of the gas turbine is the ratio of produced net work and added heat power. The efficiency can be rewritten to the following, according to Cengel (1998).

$$
\eta=\frac{w_{n e t}}{q_{\text {in }}}=1-\frac{1}{r_{p}{ }^{(\kappa-1) / \kappa}}
$$

where $r_{p}$ is the pressure ratio in the turbine and $\kappa$ is the ratio of specific heats. The higher pressure ratio of the gas turbine, the higher is its efficiency. The equation is only valid for ideal gas turbines with no friction and reversible processes. 


\subsection{The non-ideal Brayton cycle}

In reality compression and expansion are not ideal processes. Friction and turbulence cause an increase in entropy, which can be seen in the figure below. There are also pressure drops in pipes and during combustion.

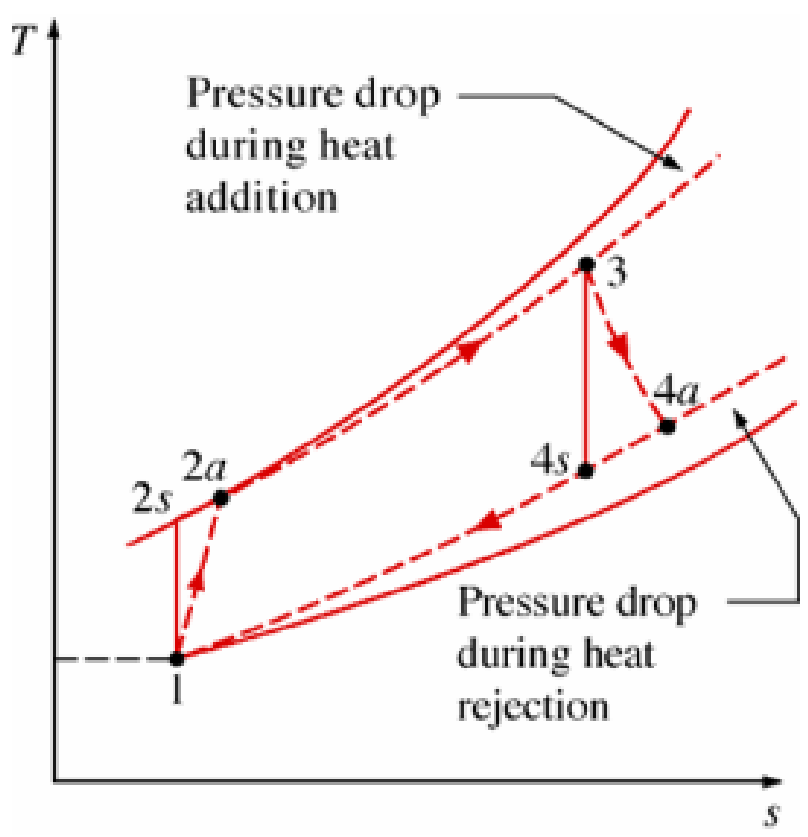

Figure 3: The deviation of an actual gas turbine from the ideal Brayton cycle as a result of irreversibilities, Cengel (1998)

\subsection{Regeneration}

There are large gains to be made if some of the heat in the exhaust gas leaving the turbine can be reused, instead of being rejected to the surroundings. This occurs in a regenerator, often called a recuperator. The hot exhaust gas can preheat the fresh air going into the combustion chamber, thus reducing the fuel requirements for the same net work output, Cengel (1998). The efficiency can now be written as:

$$
\eta=1-\left(\frac{T_{1}}{T_{3}}\right) r_{p}^{(k-1) / k}
$$

where $T_{1}$ and $T_{3}$ are the temperatures before compression (the ambient temperature) and after combustion respectively. The equation gives that the higher difference between the temperature at which heat addition (combustion) and heat rejection (exhaust gas leaves and fresh air comes in) occurs, the higher is the efficiency. The recuperator increases the combustion temperature, thus increasing the efficiency. In the T100 microturbine, the increase in efficiency at full power load, $100 \mathrm{~kW}$, is from $17 \%$ to $30 \%$ ! However, not all gas turbines can or should use a recuperator. If the temperature of the fluid at the turbine outlet is lower than at the compressor outlet, the heat flow would go in the wrong direction. This occurs in gas turbines with very high pressure ratios, since the temperature increase/decrease in the compressor/turbine depends on the pressure ratio at which they operate, see equation (5.1.8). The initial cost will also be higher and the system will be more complex and likely to failures. If the gas turbine is only used as a back-up and run for a few days a year, a recuperator might not be advantageous. 


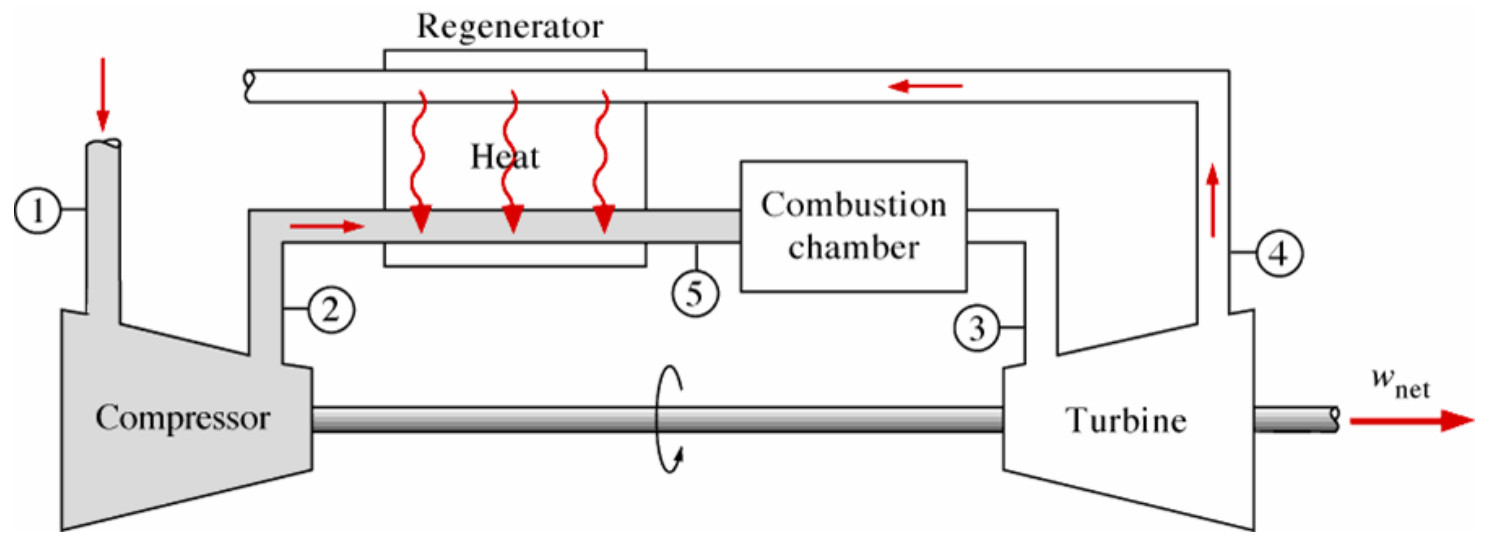

Figure 4: An ideal Brayton cycle with a regenerator, Cengel (1998)

In larger gas turbines there are additional possible stages, as for example intercooling and two stage expansion and compression. These stages will increase the total efficiency, but will make the gas turbine more expensive to manufacture and more complex. For smaller gas turbines these additional features usually do not pay off.

\section{The T100 microturbine system}

In this chapter I will describe the T100 microturbine and explain some special features that make it different from normal gas turbines.

\subsection{The T100 microturbine}

The microturbine T100 is a power generation system that is based on a combination of a small gas turbine and a directly driven high-speed generator. The generator is placed on the same shaft as the compressor and the turbine. No gearbox is therefore needed and the system uses only two bearings, reducing the friction losses to a minimum. The microturbine technology is described in Malmquist (1999).

The design of Turbec's microturbine has emphasized simplicity, small-scale, security and reliability. The pressure ratio over the compressor is low, around 4 , compared to other gas turbines. This is one reason why it is called a microturbine. Another reason is the actual physical size. The compressor and the turbine are $0.15 \mathrm{~m}$ in diameter and the entire enclosure is only $2.92 \times 1.90 \mathrm{x}$ 0.87 meters. This makes installation very easy e.g. in a normal basement. The power output $100 \mathrm{~kW}$ is chosen to fit a market demand, which corresponds to hotels, green houses, sport facilities and wastewater treatment plants. If the demand increases over time, another T100 microturbine can be installed.

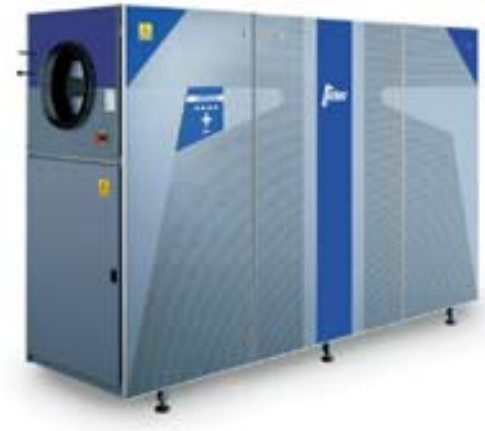

Figure 5: The T100 microturbine, (Turbec AB)

The electricity created by the high-speed generator is converted into AC voltage with a constant frequency by a power converter that is a part of the power electronics. The power electronics control the electric variables of the microturbine and the machine can readily be connected to the power grid.

The microturbine uses a combustor that normally runs on natural gas but it can be modified to accept various fuels such as diesel, kerosene, ethanol, and bio gas. 
An exhaust gas recuperator is included that improves the efficiency of the system substantially. The machine is designed to run for a long time on full load and the extra investment that the recuperator requires is quickly saved. The recuperator is also especially beneficial due to the low pressure ratio of the T100. The difference in temperature after the compressor and after the turbine is large and the recuperator can then transfer large amounts of heat from the exhaust gas to the compressed air; see the regeneration section.

There is also a gas/water heat exchanger, where water is heated by the exhaust gases coming from the recuperator. The hot gas has a heat potential that can deliver $167 \mathrm{~kW}$ at full load to the hot water system. With an electric efficiency of $30 \%$ and the extra gas/water heat exchanger, the total efficiency is about $80 \%$ ! The water can then be used to heat buildings. In countries where heat is in abundance, an absorption chiller can replace the gas/water heat exchanger to provide air conditioning. This application will shortly be introduced in the T100 microturbine.

A very important property of the T100 is its low emissions. The whole system is designed and optimized to minimize all harmful emissions. All the individual components are optimized for a certain speed or temperature where the losses are at a minimum and the efficiency at a maximum. The control system meticulously controls the speed and combustion temperature of the microturbine and keeps them constant to ensure stable and complete combustion with as low emissions as possible. The exhaust gases are actually so clean that the microturbine can be used inside green houses. In this application the T100 can supply power to the grid and heat to maintain the high temperature in the green house, while carbon dioxide in the exhaust gases are supplied to the plants as fertilizer to increase growth. In some green houses, gas boilers are used to provide the plants with heat and this extra carbon dioxide, but the demand of heat is lower than the demand of carbon dioxide so much heat is wasted. Instead some of this heat can be used to produce electricity by installing a T100 microturbine.

Below a schematic figure of the thermodynamic stages of the T100 CHP is shown. As can be seen an extra heat exchanger is used to heat water with the hot exhaust gases coming out the recuperator.

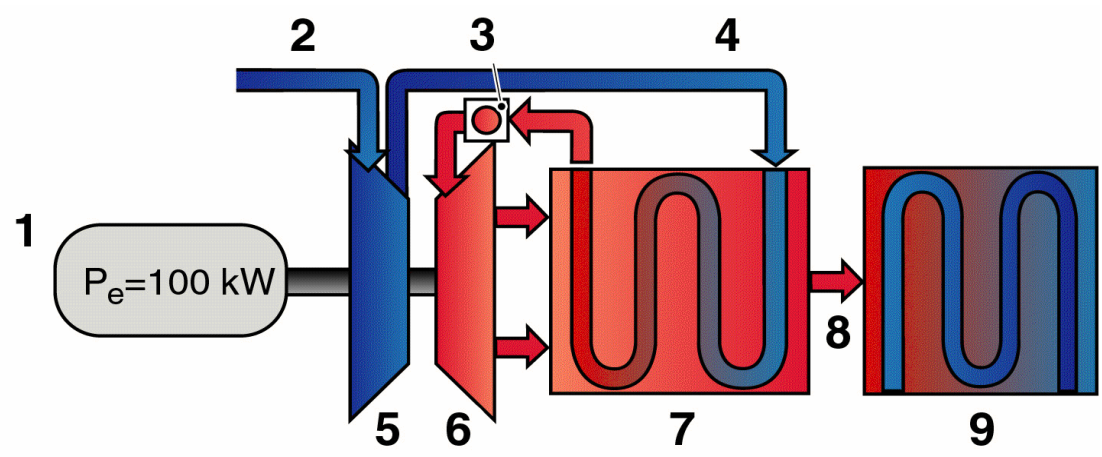
1. Generator
2. Air inlet
3. Combustor
4. Air to
recuperator
5. Compressor

6. Turbine

7. Recuperator

8. Exhaust gas to heat

exchanger

9. Gas/Water heat exchanger

Figure 6: Scheme of thermodynamic parts of the T100 microturbine, (Turbec AB) 


\subsection{Operation Modes}

The normal operation mode is the parallel mode, i.e. the microturbine produces heat and power parallel to the power grid. The local power grid is connected to an outer power grid. If the T100 produces more power than needed in the local power grid, it is exported via the outer grid. If it produces less power, then the remaining power is taken from the outer grid. The owner can decide when and how much power the microturbine should produce depending e.g. on the price of the power from the outer grid. During nights power from the outer grid is cheaper and then the microturbine can be stopped.

The other important mode of operation, not yet fully implemented and tested, is the stand-alone mode. This means that the outer power grid has been disconnected due to some reasons, e.g. black-outs, power shortages and technical failures. Then the microturbine has the sole responsibility to produce power to the local power grid. This puts very high demands on speed and reliability. The voltage and frequency needs to be kept constant regardless of the loads on the power grid. In parallel mode, the control system is slow in order to keep the thermal fluctuations in the machine to a minimum. In stand-alone mode there is no possibility to take such precautions since the power output is the primary objective.

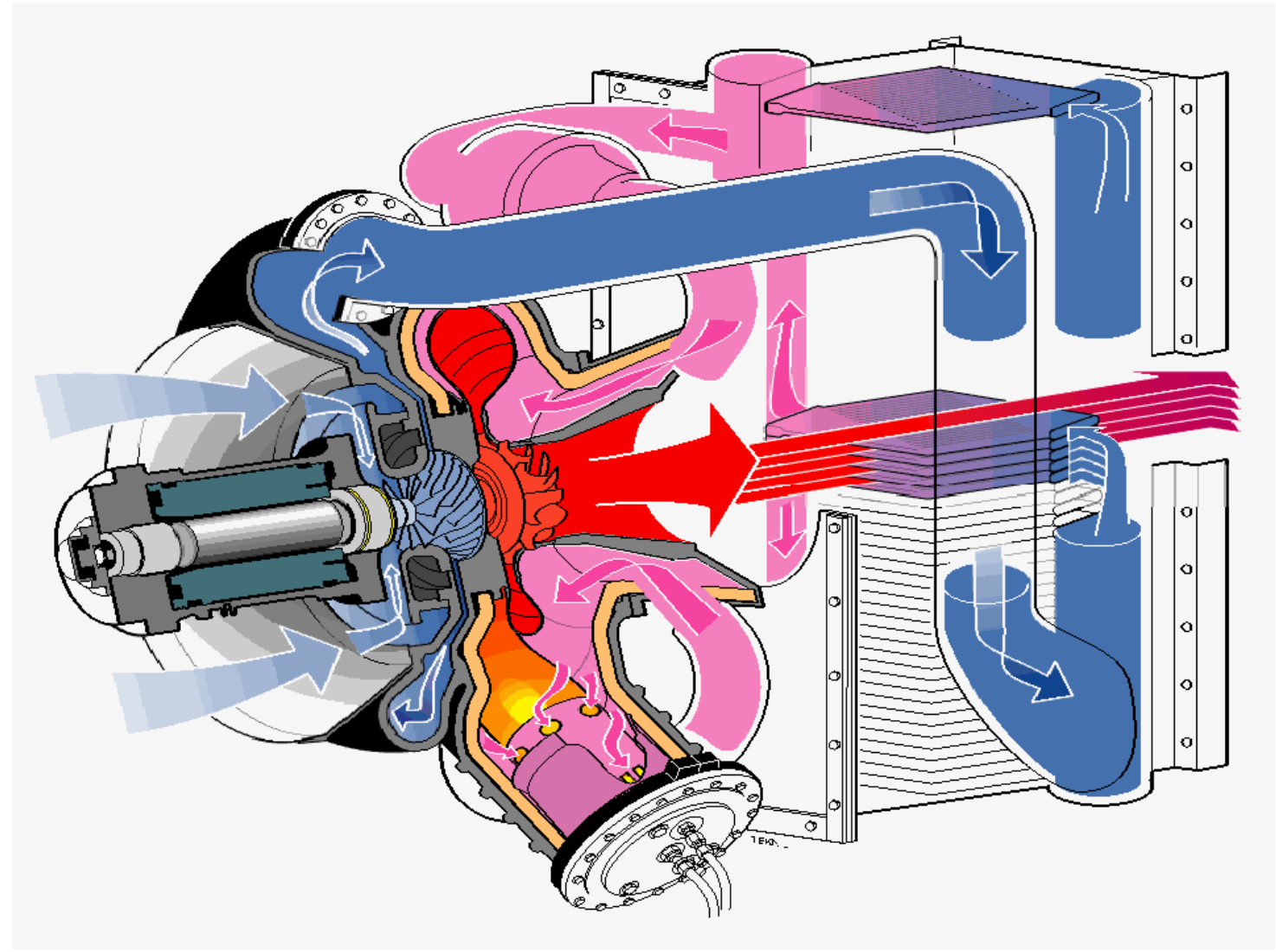

Figure 7: A flow model of the T100 microturbine, notice the single shaft that is the only rotating part, (Turbec AB) 


\subsection{Extra dynamics}

A gas turbine consists of different components and when all components are put together some extra dynamics arise. The flow model of the T100 in figure 7 above gives a more detailed view of how the turbine works. Air is taken from the outside and flows around the generator into the compressor in axial direction. The air is compressed and leaves in radial direction. The highpressure air (blue pipes) is fed through the recuperator where it is preheated with the exhaust gas. Now at a much higher temperature (pink pipes) it is mixed with the fuel gas in the combustion chamber and burned. The flue gas enters the turbine radially and leaves in an axial direction (red pipes). The flue gas exchanges heat with the colder air in the recuperator and then leaves the figure. The gas/water heat exchanger (not shown in the figure) is placed directly after the recuperator.

In an ideal machine the turbine would be a purely algebraic component with no dynamics involved. Instead, when we include the turbine rotor and the turbine diffuser (the part directly after the turbine marked red in the figure above), we get some extra dynamics. The turbine rotor can store thermal energy and when the temperature of the exhaust gas decreases, due to a load change, the rotor acts as small thermal reservoir with a limited amount of energy. The rotor speed is very high, so that the energy stored in the rotor is easily transferred to the gas, thus acting as a filter on the temperature dynamics. Due to the geometric design, the turbine diffuser serves as an extra heat exchanger, although a very poor one. The extra heat exchanger has a considerably smaller effect than the recuperator, but along with the turbine rotor they have an interesting impact on the dynamics. The actual effect can be seen in the verification section.

\subsection{Bypass}

The T100 CHP microturbine produces heat through the gas/water heat exchanger. The amount of heat produced is directly linked to the amount of electricity produced. In some cases the owner wants to produce less heat than electricity. If too much heat is transferred to the water, the water might boil and evaporate causing damage to the heat exchanger. Therefore the amount of heat transfer must be controlled in a way that does not interfere with the electricity production. The solution is a bypass system, where some or all exhaust gases are diverted around the gas/water heat exchanger in a similarly way as a valve works. Due to time and the low priority given to bypass operations, there is no complete verified model of the bypass function in this thesis.

\section{Simulation Tools}

In this chapter I will describe the tools I have used in the modelling of the microturbine. There are different layers of tools. First there is the simulation language, the language the model is written in, e.g. Modelica. Then there are libraries e.g. ThermoFluid that contain complete submodels. And last there is a simulation program e.g. Dymola, that contains a graphical user interface, different solvers, plot functions and parameter settings.

\subsection{Selecting the tools}

Thermodynamic models are often large and complex. They consist of volumes, flows but also electronic components. There exist numerous simulation languages and programs e.g. Fortran, Matlab/Simulink and Modelica/Dymola to just mention a few of them. To make things more complicated there are several different simulation programs used within Turbec AB for different purposes and applications. For static simulations, a program called Dynamic Systems Analyzer v2.0 (DSA) is used. DSA is a program developed at Volvo Aero Corporation AB. With version 2.0 only static simulations are possible. A very detailed model of the T100 with all components 
and connections is used in simulations to get static results with high accuracy. The model has then been verified with experimental data and calibrated to further improve the model's accuracy.

Matlab/Simulink is used to model the power electronics and control system including a coarse thermodynamic model. The problem with Matlab/Simulink is that the program does not model flows well in general and flows in sudden changing directions in particular. Matlab/Simulink supports causal modelling with fixed causality of inputs and outputs and hard work is needed to put the system in the order of an ordinary differential equation (ODE). The modelling is also not component-oriented. More discussions can be found in the Modelica chapter. A third program, ICES, (Internal Combustion Engine Simulation), is used to create a dynamic model of the microturbine engine. The program is written in Fortran and is component-oriented. The drawback is that Fortran does not have a graphical user interface, which makes it harder to learn. Most models are however written in Fortran and it is the most widely used modelling language in the industry. The very useful library ThermoFluid is also a major incitement to choose Modelica as the modelling language. Fortran does have a similar library for thermodynamic models but in other areas as e.g. electronics Modelica is better situated than Fortran. Models in Modelica can also be transferred to other simulation environments as S-functions in Matlab/Simulink, which ICES cannot. It is possible to execute Fortran and C code in Matlab but to integrate the model with other Simulink models you need to convert the code into S-functions. In total this lead us to choose Modelica as our choice of modelling language with ThermoFluid as a prime resource for the basic blocks. In choosing Modelica we also chose Dymola as our simulation program since those two are closely linked. The company behind Dymola, Dynasim AB is also very active in the development of Modelica as the new language of modelling. After this initial discussion I will describe the different simulation tools used in this thesis.

\subsection{Dymola}

Dymola, Dynamic Modeling Laboratory, is a simulation program consisting of a compiler, graphical user interface, numerical solvers and plot functions. For more information on Dymola, see the Dymola manual (2001) or their home page www.dynasim.se.

The models are written in the Modelica language. Dymola generates the $\mathrm{C}$ code, which is compiled and linked with the solver routines into a simulation executable, Dymosim. In the Dymola Main Window parameters, initial conditions and simulation settings can be set. 


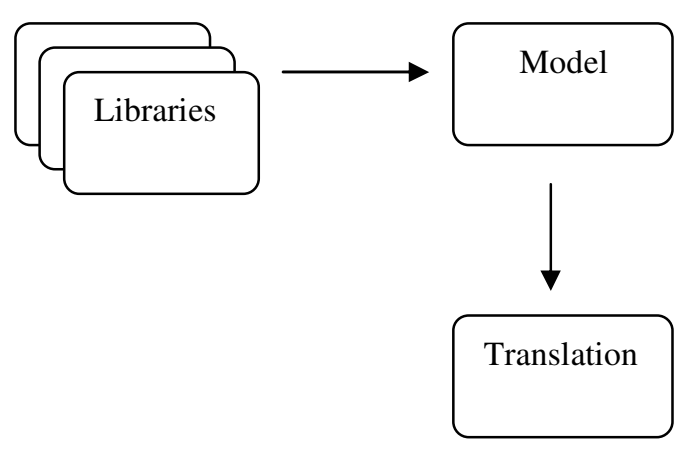

Model Editor (code)

- Model composition

- Default parameter setting

- Definition of equations

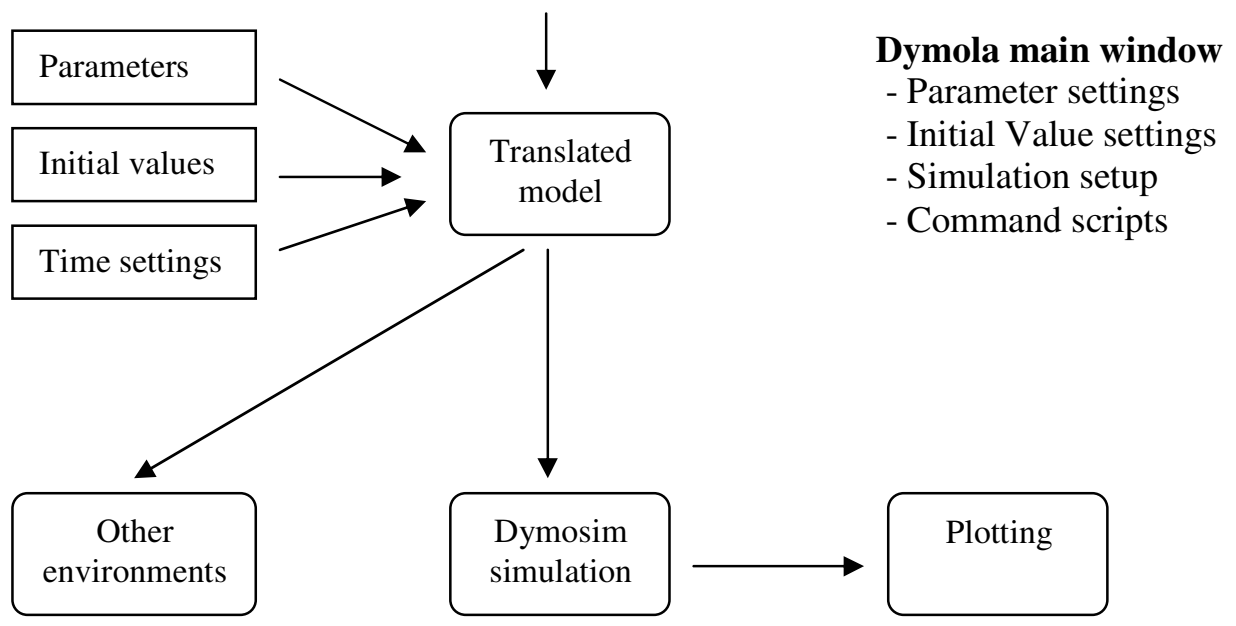

Figure 8: The Dymola modeling environment, (Dynasim AB)

Command scripts are small text scripts where common commands can be written, saved and then executed all together, every time the script is run (similar to an m-file in Matlab).

The models are composed of several small blocks representing physical things like pipes, compressors and turbines. Each block has its equations governing the states of the block and the algebraic equations. When combining these blocks the equations must also be combined, but there are different options on how to do it.

In Matlab/Simulink it is necessary to rewrite all equations according to one fixed flow order as ordinary differential equations (ODE). The equations can also be said to be in state-space form:

$$
\dot{x}=f(x)
$$

The system has well-defined inputs, outputs and states. This is a non-trivial exercise and often time-consuming. The equations also tend to lose their specific physical representation.

Another option is to use non-causal modelling, which means that the equations are written in a natural form as a system of differential-algebraic equations:

$$
f(x, \dot{x}, y)=0
$$

where $x$ is the vector of unknowns that appear differentiated in the equation and $y$ is the vector of unknowns that do not appear differentiated. Dymola has a powerful tool that automatically rewrites the equations to the desired ODE form. In this way the equations can be kept in separated blocks, so that the physical representation remains. A drawback with this method is that it gets 
harder to understand the dependency of each variable and in what order they are calculated. The differences between the two methods can be seen in the following example:

A motor drive is to be simulated and in Modelica each block can be assembled into the complete drive. Each block has its equations and connections to the surrounding blocks. It is very simple to understand what every block correspond to in the real world.

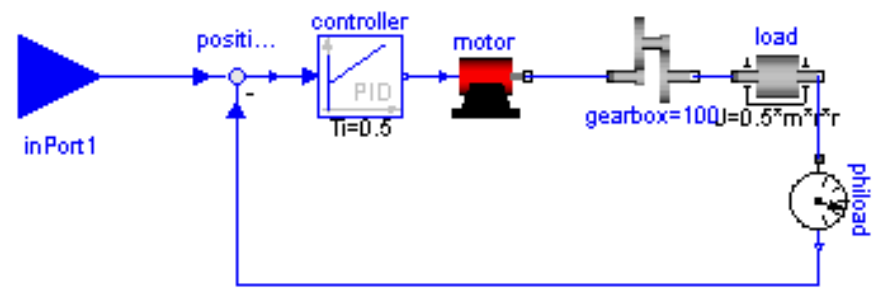

Figure 9: A motor drive modelled in Modelica, (Dynasim AB)

In Matlab/Simulink one cannot simply put the same blocks together. The equations need to rewritten manually and the model can then be constructed using new blocks, not necessarily with any physical representation, just like the gain block in the figure below. The gain block consists of the gearbox and the inertias of the motor and the load.

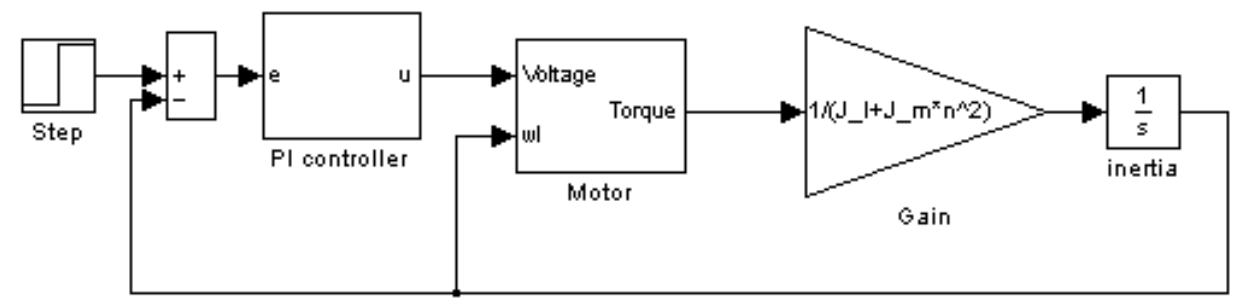

Figure 10: A motor drive modelled in Matlab/Simulink, (Dynasim AB)

\subsection{Modelica}

Modelica is an object-oriented language designed to allow convenient, component-oriented modelling of complex heterogeneous physical systems. Important parts of Modelica are the object-oriented structure and the non-causal modelling, which has been discussed in the Dymola section. For more detailed information see Modelica home page www.modelica.org. A free Modelica editor is available at http://www.et.dtu.dk/fme.

\subsection{The ThermoFluid Library}

This library is developed at the department of Automatic Control at Lund Institute of Technology. The main purpose is to provide a general framework and basic building blocks for modelling thermo-hydraulic systems, written in the Modelica language. More information about the library can be found in Tummescheit (2002) or any of the references of Eborn et al.

To provide a general and flexible framework, a library must emphasize the small blocks on the behalf of the more complex specific systems like a power plant. The basic design principles are as follows: 
1. One unified library both for lumped and distributed parameter models,

2. separation of the medium models, which can be selected through class parameters,

3. both bi- and unidirectional flows are supported and

4. assumptions (e.g. if gravity influence should be modelled) can be selected by the user from user inputs.

The three major atomic parts of the library are:

Control Volumes (CV) have a finite volume and are storages for mass and energy. The CV can be either lumped or discretisised in space.

Lumped Flow Models (FM) are the results of a modelling abstraction, where the volume is neglected, e.g. in valves and compressors. Algebraic equations relate variables, e.g. the pressure drop and mass flow.

Dynamic Flow Models (also abbreviated FM) can also calculate the storage of momentum in a control volume.

In combination with thermal models, dynamic flow models are only used when the focus is on very fast transients like emergency shutdowns and the change of momentum is of importance. The lumped flow models are used, when the emphasis is on slow thermal applications, as e.g. the temperature in heat exchangers. In the model of the T100 microturbine lumped flow models are used, since the main issue is normal power production and the corresponding thermal variables.

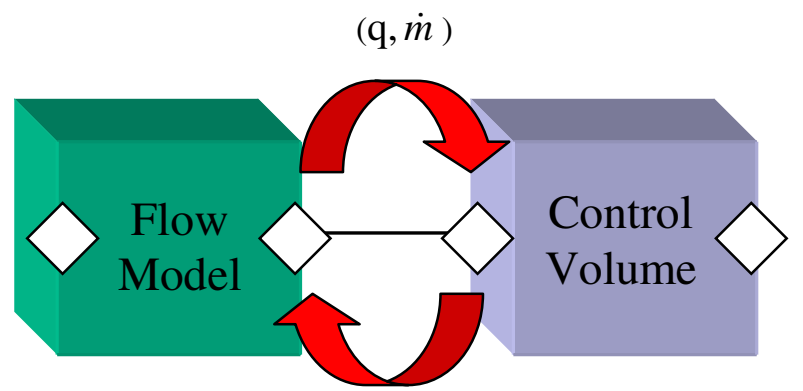

$$
(p, h, \rho, T, s, \kappa)
$$

Figure 11: Interaction between control volume and flow model with flow connectors, Perez (2001).

Between the atomic parts, there are flow connectors (the diamond shape blocks in figure 11). To be able to evaluate the thermodynamic variables it is necessary to alternate control volumes and flow models after each other. A complete description of the sequence of calculation will be given in the end of this chapter. To fully understand the model and how to use it, it is also essential to understand what the connectors really show and where its properties come from. All values of the transported properties in a control volume are mean values for the whole control volume. This means that the temperature at the inflow connector of the $\mathrm{CV}$ is the same as at the outflow connector, i.e. the mean value of the whole control volume. As mentioned above, this holds for all transported properties like density, enthalpy and entropy etc. In order to get the true temperature of the inflow side of the control volume we have to use the flow variables $\dot{m}$ and $q_{\text {conv }}$. A misunderstanding can easily be made; at the outflow connector of a flow model, the temperature shown is the mean temperature of the following control volume, not the actual temperature, which is hidden in the mentioned flow variables. 


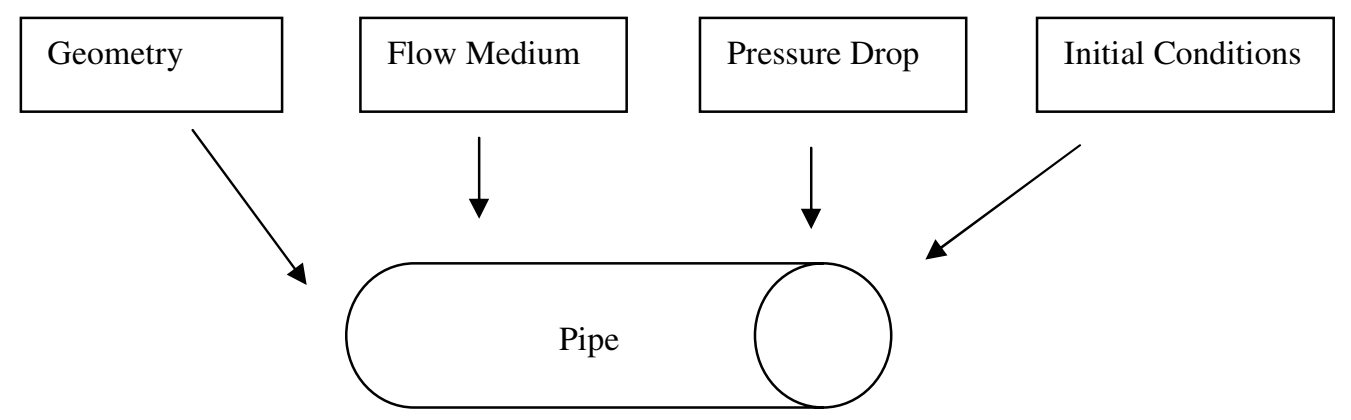

Figure 12: A pipe model inherits code concerning its properties from several classes

As an example of multiple inheritance, a schematic figure of a pipe model is shown in figure 12 above. The pipe model, which consists of a control volume and a flow model, inherits code from among others the classes Geometry, Medium, PressureDrop and Initialization. Then any change in these classes will immediately take place in the pipe as well.

The use of multiple inheritance creates a powerful flexibility when important properties can be separated as e.g. slow and fast dynamics, medium models, choices of dynamic states, heat transfer and pressure drop. This makes the separate properties of the model very easy to replace, but the code becomes less readable, since the corresponding equations can then be found in many different files. Dymola can automatically generate html-documentation of each model with hypertext links to all inherited classes, which helps to overview the model structure.

The medium models in ThermoFluid are carefully chosen to be very accurate but yet fast for computational reasons. The enthalpy, heat capacity and entropy for ideal gases are given below as functions of temperature, taken from Gordon (1994).

$$
\begin{gathered}
h(T)=R\left(-a_{1} T^{-1}+a_{2} \ln T+a_{3} T+a_{4} \frac{T^{2}}{2}+a_{5} \frac{T^{3}}{3}+a_{6} \frac{T^{4}}{4}+a_{7} \frac{T^{5}}{5}+b_{1}\right) \\
c_{p}(T)=R\left(a_{1} T^{-2}+a_{2} T^{-1}+a_{3}+a_{4} T+a_{5} T^{2}+a_{6} T^{3}+a_{7} T^{4}\right) \\
s(T)=R\left(-a_{1} \frac{T^{-2}}{2}-a_{2} T^{-1}+a_{3} \ln T+a_{4} T+a_{5} \frac{T^{2}}{2}+a_{6} \frac{T^{3}}{3}+a_{7} \frac{T^{4}}{4}+b_{2}\right)
\end{gathered}
$$

For each medium the coefficients $a_{\mathrm{i}}$ and $b_{\mathrm{i}}$ and other parameters are given in a data record. Water/steam data is taken from IAPWS-IF97, Wagner (1998).

The ThermoFluid library uses non-causal modelling, which means that the simulation program, in this case Dymola, takes the given equations and automatically turns it into a causal sequence of calculation for the solver to use it. For the user to understand the sequence of calculation, the algorithm will now be further explained, Perez (2001). 


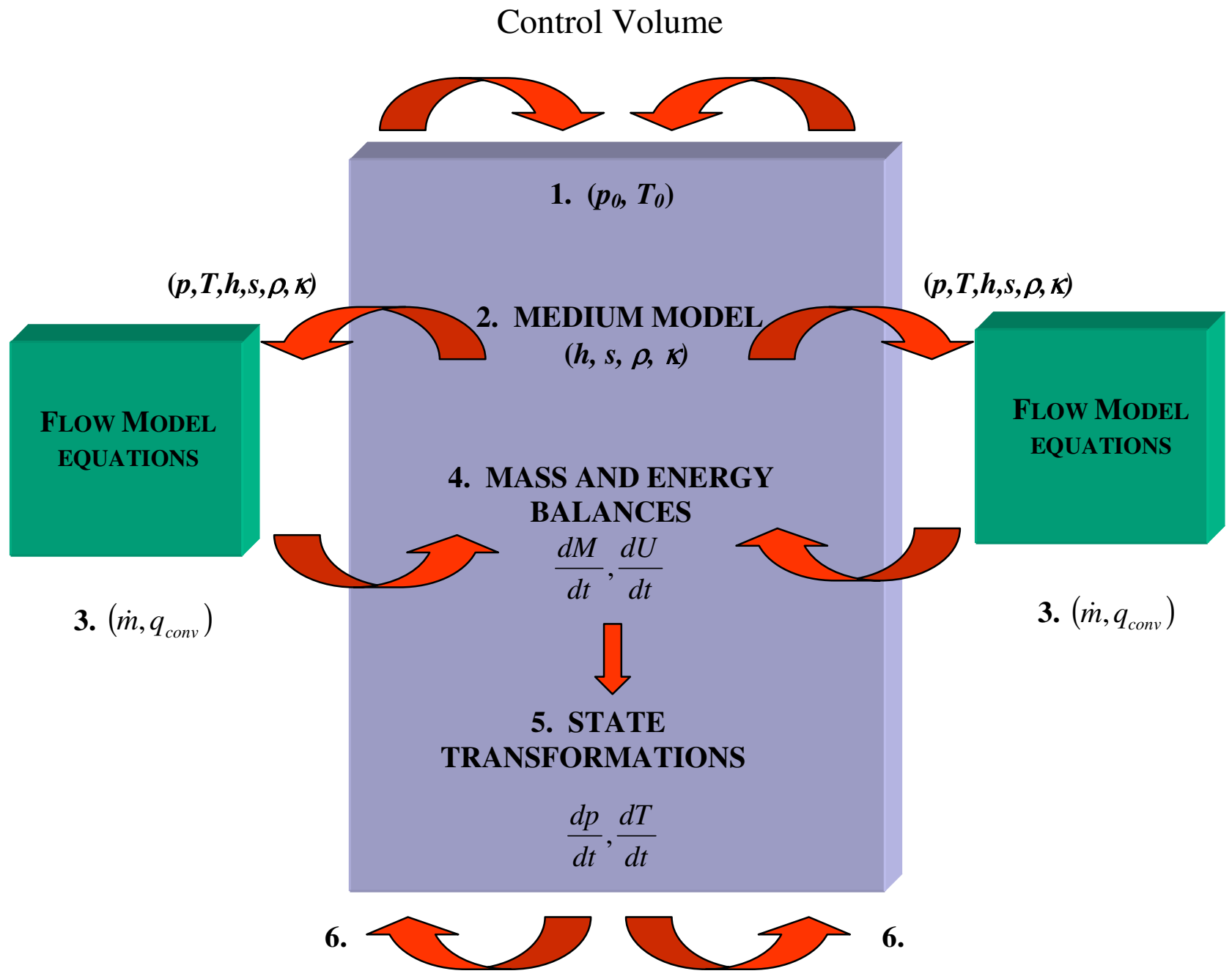

Figure 13: Sequence of computation in ThermoFluid, Perez (2001)

In this example, pressure and temperature are the chosen states, but there are also other options as e.g. temperature and mass fraction or temperature and mass. The green flow models in the figure above interact with another control volume on the left and right side respectively. The whole model is a continuous chain of control volumes and flow models in alternating sequence.

1. The user has to set some initial pressure and temperature for each control volume or provide equations that will calculate them.

2. Knowing the two parameters, the medium models are used to evaluate all other thermodynamic variables, like $h, d, s$ and $\kappa$ in the control volume.

3. The variables from the control volumes are used in the flow connectors to calculate the new mass and energy flows in the adjacent flow models.

4. The mass and energy flows from the adjacent flow models to the control volume are then used to calculate the mass and energy balances in the control volume in form of the time derivatives $\mathrm{dM} / \mathrm{dt}$ and $\mathrm{dU} / \mathrm{dt}$.

5. A class called StateTransformation transforms these derivatives to the time derivatives of the states, pressure and temperature, i.e. $\mathrm{dp} / \mathrm{dt}$ and $\mathrm{dT} / \mathrm{dt}$. 
6. Finally, these time derivatives are used to evaluate the new values of the states. Now the temperature and pressure is once again known and the sequence returns to number 2 .

\section{Thermodynamic theory and modelling}

In this chapter I will describe the individual components of a gas turbine, the thermodynamic theory of the components and how I have chosen to model them. The models of the compressor, turbine and combustion chamber are originally taken from Perez (2001) and have then been modified. Some of the following figures that describe vital parts of the microturbine, lack axes and are rescaled because of the classified information they contain.

\subsection{The compressor equations}

A centrifugal compressor is designed to increase the pressure of the gas using rotation. It uses mechanical work to rotate the rotor, thus accelerating the gas. After the rotor the gas passes a diffuser, where the increase in cross-section area gives a decrease in velocity and an increase in pressure, according to Bernoulli's law. The total energy equation below can be found in Cengel (1998).

$$
d q-d w-d h-d k e-d p e=0
$$

where $d q$ is the specific external heating, $d w$ is the specific net work, $d h$ is the change in specific enthalpy, $d k e$ is the change in kinetic energy and $d p e$ is the change in potential energy. Here the term specific means the amount per unit mass. We can assume that the compressor is adiabatic (i.e. no heat flow in or out) so we can neglect the term $d q$. We do not have any height differences so the same goes for the term $d p e$. The velocity does not change very much from the inlet of the compressor to the exit of the diffuser. This small change in kinetic energy can therefore be neglected. This leaves us with:

$$
-d w=d h
$$

The temperature and therefore the enthalpy increase at compression, resulting in $d h$ as a positive number. The specific heat is defined as the energy required to raise the temperature of a unit mass of a substance by one degree at constant pressure and can be written as:

$$
c_{p}(T)=\left(\frac{\partial h}{\partial T}\right)_{p}
$$

After integrating over the compressor we get:

$$
-w=\int_{1}^{2} c_{p}(T) d T
$$

where the subscripts 1 and 2 denote the inlet and the outlet respectively. The minus sign in front of $w$ indicates that work is done on the gas from the surroundings, i.e. the compressor. The specific heat can be very accurately calculated with equation (4.4.2), but in this derivation it is enough to use the approximation of constant specific heat. The specific heat for air and water vapor is plotted in figure 14 . 


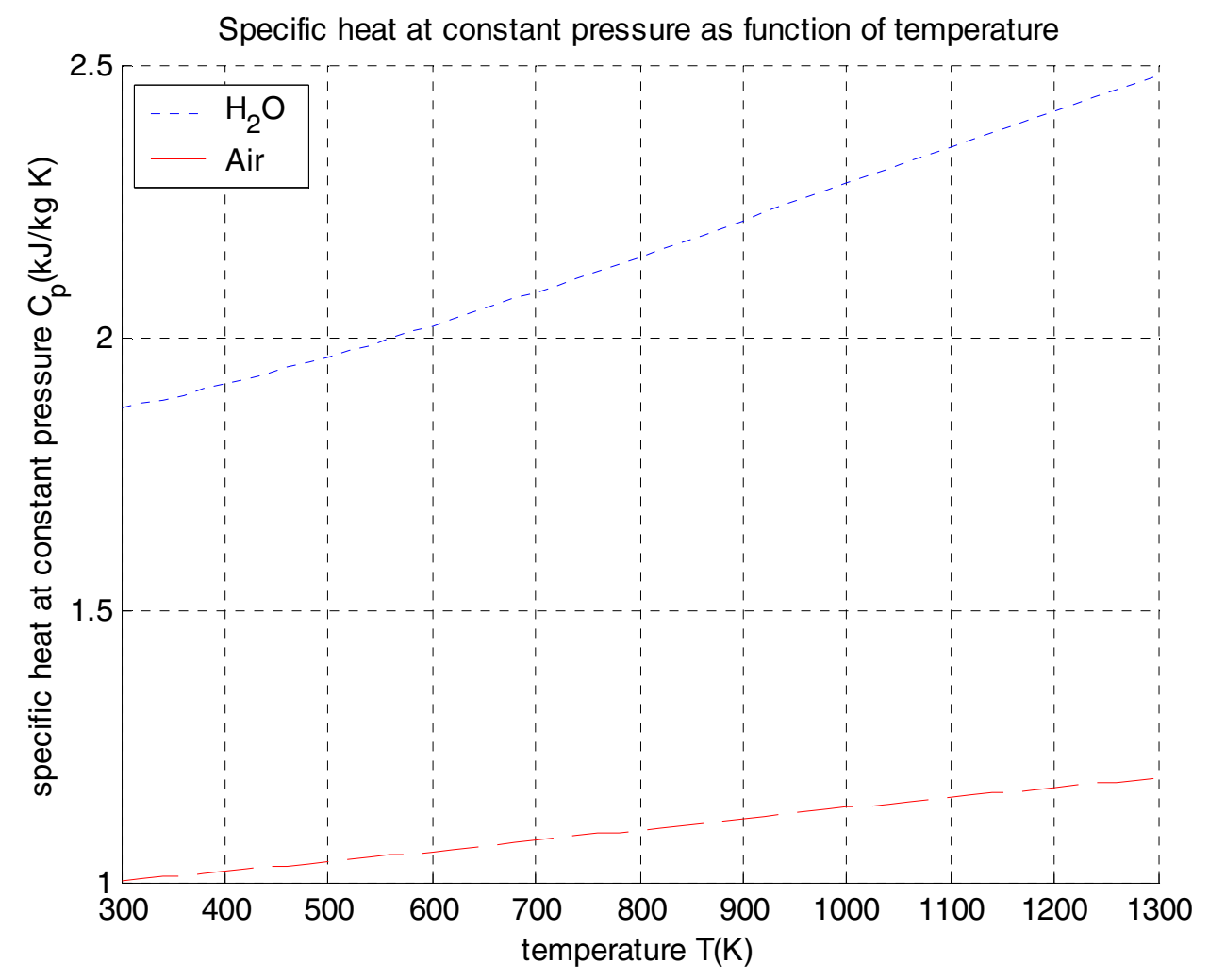

Figure 14: Specific heat capacity for air (dashed red) and water vapor (dotted blue), Cengel (1998).

Note in figure 14, the larger heat capacity of water vapor, which makes the mass percentage of water vapor in air an important variable. When we use a constant specific heat, we use the average of the value at the inlet and the outlet of the compressor. Integration gives:

$$
-w=c_{p, a v g}\left(T_{2}-T_{1}\right)=c_{p, a v g} T_{1}\left(\frac{T_{2}}{T_{1}}-1\right)
$$

Another way to calculate $c_{p}$ is taken from Philip (1999):

$$
c_{p}=\left(\frac{\kappa}{\kappa-1}\right) R
$$

where $\kappa$ (sometimes denoted $k$ ) is the ratio of the specific heat at constant pressure and constant volume. $R$ is the gas constant for the specified gas, in this case air. Note that $\kappa$ also varies with temperature, but not as much as $c_{p}$, since the variations of $c_{p}$ and $c_{v}$, due to temperature, almost cancel each other. The value of $\kappa$ is taken as the average of the inlet and the outlet of the compressor, but the subscript is from now on dropped. Then our equation for the work can be rewritten as:

$$
-w=\left(\frac{\kappa}{\kappa-1}\right) R T_{1}\left(\frac{T_{2}}{T_{1}}-1\right)
$$

Of the parameters above, only $T_{2}$ varies, since $T_{1}$ is the inlet temperature and $R$ and $\kappa$ are approximately constant. The work is at minimum, when $T_{2}$ is minimal. This is achieved at isentropic compression. Isentropic means that the process is adiabatic (no heat flows in or out of the system) and reversible (the process can be reversed without losing energy, e.g. no friction). 
From Cengel (1998) we get the following expression for isentropic compression where the subscript is denotes the variable at isentropic conditions:

$$
\frac{T_{2, i s}}{T_{1}}=\left(\frac{p_{2}}{p_{1}}\right)^{\frac{\kappa-1}{\kappa}}
$$

The equation for the specific work can now be calculated using the inlet and outlet pressure and input temperatures as follows:

$$
-w_{i s}=\left(\frac{\kappa}{\kappa-1}\right) R T_{1}\left(\left(\frac{p_{2}}{p_{1}}\right)^{\frac{\kappa-1}{\kappa}}-1\right)
$$

This is only valid for ideal processes, processes that are adiabatic and reversible. To compensate for actual conditions, we use the thermodynamic variable, isentropic efficiency, defined as the ratio of the enthalpy change at isentropic conditions and the actual enthalpy change:

$$
\eta_{i s}=\frac{h_{2, i s}-h_{1}}{h_{2}-h_{1}}=\frac{c_{p}\left(T_{2, i s}-T_{1}\right)}{c_{p}\left(T_{2}-T_{1}\right)}=\frac{T_{2, i s}-T_{1}}{T_{2}-T_{1}}
$$

The value of the isentropic efficiency can be determined through experiments, where the temperatures are measured. Results from tests show that the efficiency does not however remain constant for all pressure ratios. For increasing pressure ratios, there is a decrease in isentropic efficiency. A physical explanation is that the increase in temperature due to friction in one stage of the compression results in more work being required in the next stage, Cohen, (1996). Apart from friction, there is another factor that contributes to a lower efficiency. If another speed is used, different from the rotational speed the compressor is optimally designed for, this can cause a difference in alignment with the gas flow and the impeller vanes. If the flow enters in an angle different from the angle of the impeller vanes, there will be small turbulent eddies right behind the vanes and the efficiency of the compressor will decrease.

The actual specific work required can be formulated as:

$$
-w=\left(\frac{\kappa}{\kappa-1}\right) R T_{1}\left(\left(\frac{p_{2}}{p_{1}}\right)^{\frac{\kappa-1}{\kappa}}-1\right) \frac{1}{\eta_{i s}}
$$

The actual power needed to run the compressor is then simply:

$$
P_{\text {compressor }}=\dot{m} \cdot w
$$

There can also be mechanical losses in the compressor. The compressor is mounted on a single shaft and the relation between torque and power can be written as:

$$
P_{\text {compressor }} \cdot \eta_{\text {mec }}=\tau_{\text {compressor }} \cdot \omega
$$

where $\omega$ is the angular velocity of the shaft, $\tau$ is the torque the compressor consumes and $\eta_{\text {mec }}$ is the mechanical efficiency of the compressor.

\subsection{The compressor model in Modelica}

Based on the equations derived above a model of the compressor was developed. The model is taken from Perez (2001) and then modified.

There are some properties that cannot be calculated analytically, e.g. the isentropic efficiency and the mass flow through the compressor. These must instead come from empirical data, which is given in the form of a compressor map. In the map, the mass flow and efficiency are given as functions of other known variables, usually the speed and the pressure ratio. The compressor map 
can be generated either by physical experiments or very detailed calculations. In the case of the T100, the computations were carried out at Volvo Aero Corporation (VAC) AB.

The map is generated from a steady state situation for a certain temperature, pressure and speed. In order for the map to clearer present its data, a usual trick is to reduce the number of parameters, by using non-dimensional analysis. This is done in the form of the non-dimensional variables, corrected speed and corrected mass flow. The variables are normalized with the ambient temperature and pressure during the experiment/calculation. For more background on non-dimensional analysis see Fox (1998) and Cohen (1996). The corrected speed and mass flow should also include the diameter $\mathrm{D}$ and the gas constant $\mathrm{R}$ in order to be dimensionless, but these variables are constant for the compressor, thus they can be neglected.

We define:

Pressure ratio $p r=\frac{p_{2}}{p_{1}}$

Corrected mass flow $\dot{m}_{\text {corr }}=\frac{\dot{m} \sqrt{T_{1}}}{p_{1}}$

Corrected speed $n_{\text {corr }}=\frac{n}{\sqrt{T_{1}}}$

In the figure below we can see the compressor map plotted from data computed by Volvo Aero Corporation $\mathrm{AB}$. For confidentiality, the axes of the figure are removed and the data rescaled. The characteristic shape of the map is, however, still the same.

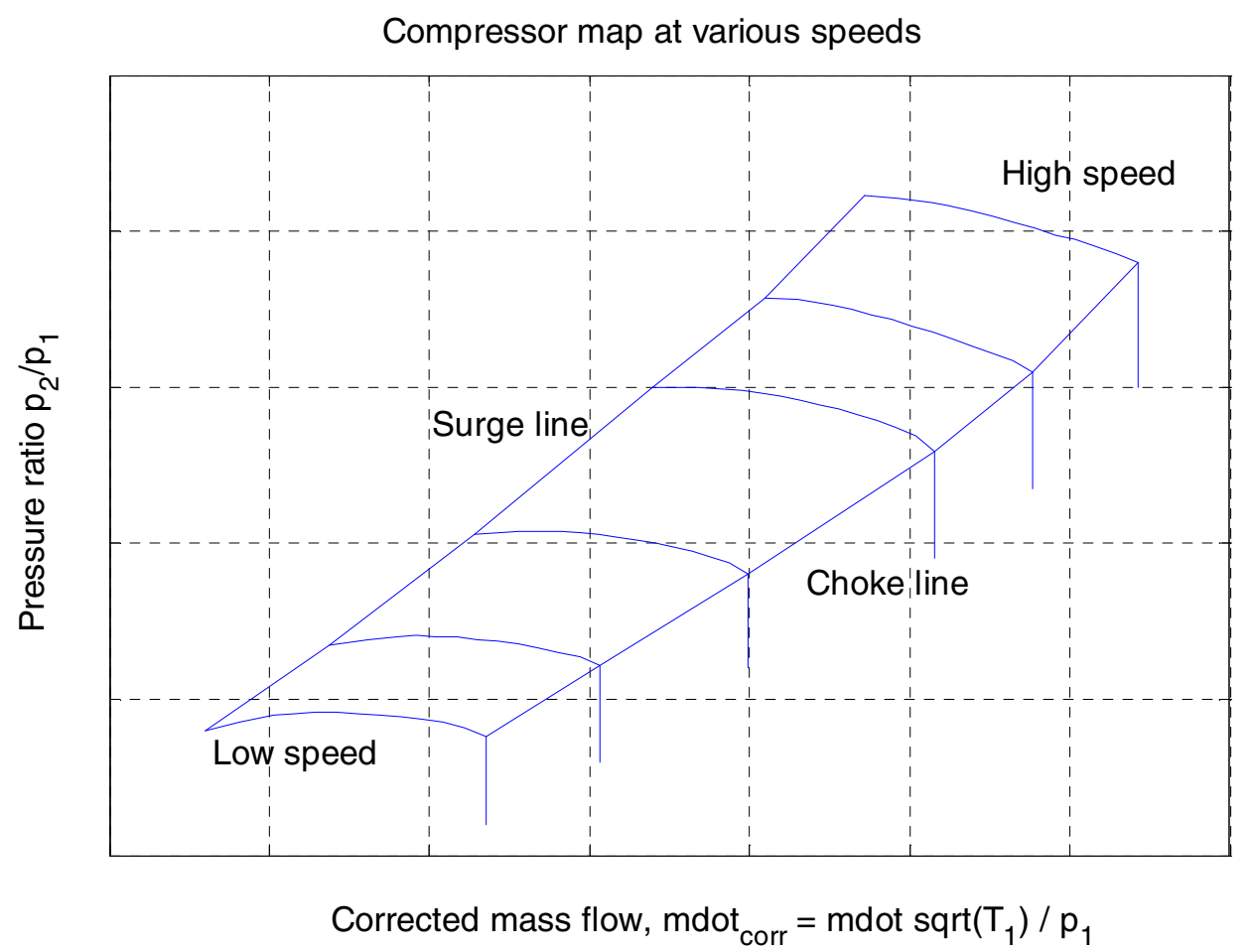

Figure 15: The compressor map generated by Volvo Aero Corporation AB 
Each of the horizontal curves corresponds to one constant speed of rotation. The range is from 20 $000 \mathrm{rpm}$ up to $74000 \mathrm{rpm}$. The area enclosed by the surge line and the choke line is the normal operating range for the compressor. On the left side of the surge line the pressure ratio decreases for decreasing mass flow, i.e. a positive pressure gradient. This can, for the lower speeds, already be seen on the immediate right side of the surge line. Surge is associated with a drop in the pressure ratio, i.e. the delivery pressure, which can lead to pulsations in the mass flow and even reverse it. It can cause considerable damage to the compressor, e.g. blade failure. The surge phenomenon is similar to wing stall of an airplane. Rotating stall is another dangerous event. It occurs when cells of separated flow form and block a segment of the compressor rotor. Performance is decreased and the rotor might be unbalanced, also leading to failures, Fox (1998).

As the pressure ratio decreases and mass flow increases, the radial velocity of the flow must also increase, in order for the compressor to blow enough gas through it. At some point the compressor cannot accelerate the flow to high enough radial velocity for the given motor speed, then maximum mass flow is reached and choking is said to occur, Cohen (1996).

The compressor map model must in one way or another represent the theoretical compressor map. One obvious method is taking the raw tables of data that produce the curves above and use them in a look-up table. Between data points bilinear interpolation can be used. This method was tested in the compressor model for the mass flow and the efficiency. Unfortunately it did not work, due to numerical problems, which will be discussed in the end of the compressor section.

Another option is to fit continuous functions to the different curves and parameterize them so that the whole map can be continuously represented, even for different speeds. This has been done in Gustafsson (1998). The curves in the map above can be viewed as ellipsoid curves and can be represented with an ellipsoid equation:

$$
\left(\frac{x}{a}\right)^{z}+\left(\frac{y}{b}\right)^{z}=c
$$

When the parameters $a, b, c$ and $z$ are varied, the form of the ellipsoid curve can be adjusted to fit any data curve from figure 15 . The parameter $a$ corresponds to the corrected mass flow at pressure ratio one (where the curve would cross the x-axis). Similarly the parameter $b$ represents the pressure ratio at zero mass flow (where the curve would cross the y-axis). The parameter $c$ is just an arbitrary constant usually taken to one. The parameter $z$ represents the curvature of the curve. 


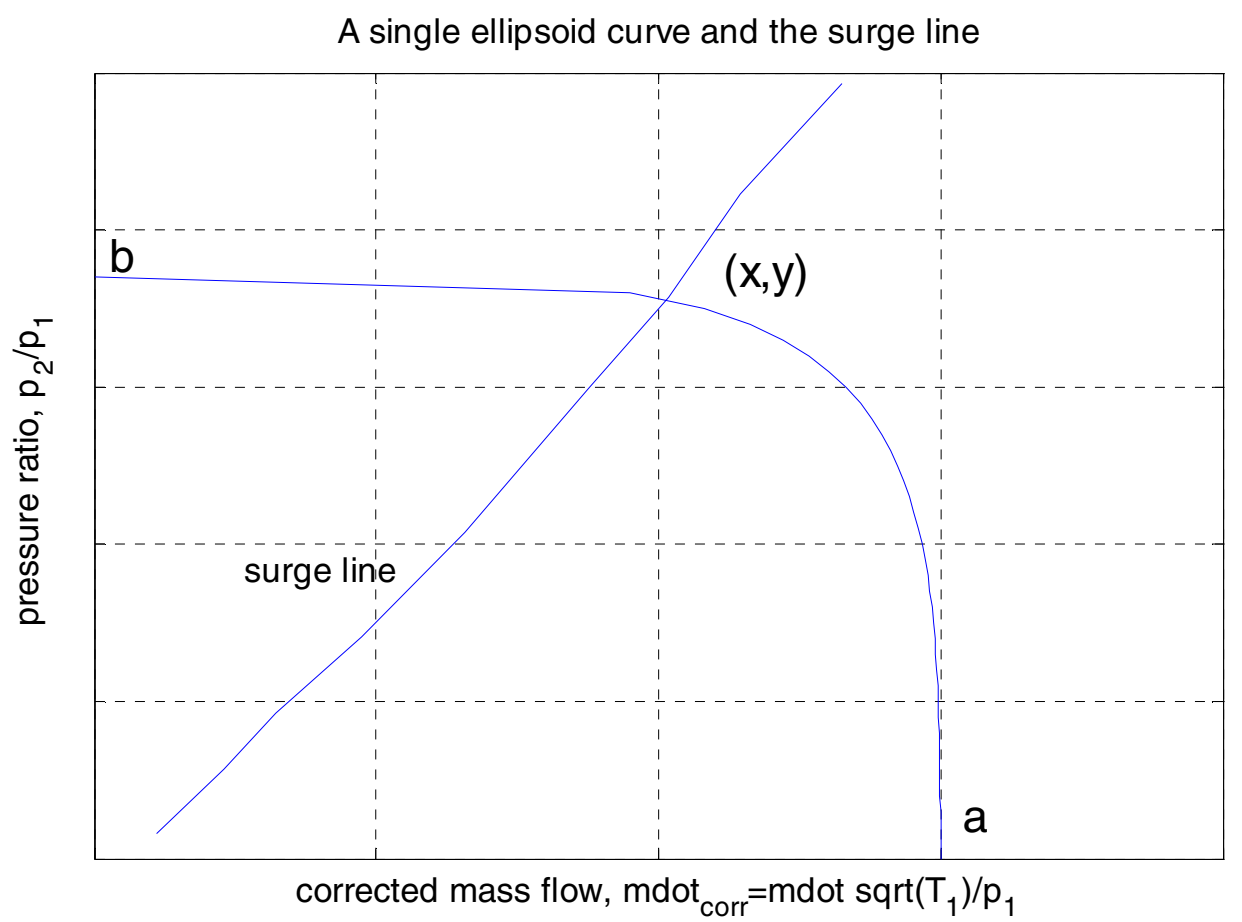

Figure 16: An ellipsoid curve based on equation (5.2.4)

The values of the $a$ parameters are first taken from the compressor map and the parameter $z$ is at first parameterized with a linear increase with speed, as done in Perez (2001). To get the $b$ parameter we use the ellipsoid equation with a known input, the point $(x, y)$ from the map, where the curve crosses the surge line. With the values of $x$ and $y$ we can calculate $b$. Now the ellipsoid curve is matched with the given data at least in one point. Unfortunately the values of $a$ and $z$ are not good enough to give accurate results all along the curve. Instead manually adjustments are made to the parameters $a$ and $z$ for each speed to minimize (via visual inspection) the deviations between the ellipsoid curves and data from the map. There is no need for the ellipsoid curves to fit outside the actual operating range; instead emphasis is put on matching the data as good as possible within the operating range. As can be seen in figure 17 below, the model curves differ a lot from the data outside this range. Now each speed from the map is modelled by equation (5.2.4), uniquely determined by its parameters $a, b, c$ and $z$.

In order to generate a continuous mapping for all possible speeds, a polynomial function is fitted for each parameter $a, b$ and $z$. The calculations were done in Matlab using the polyfit command. The polynomials are of an order between 4 and 7, depending on the nature of the data. With the polynomials, the parameters can during the simulation be evaluated for a continuous set of speeds from $21000 \mathrm{rpm}$ to 74000 rpm like this:

$$
a=\mathrm{pa}(1) \cdot \mathrm{ncorr}^{4}+\mathrm{pa}(2) \cdot \mathrm{ncorr}^{3}+\mathrm{pa}(3) \cdot \mathrm{ncorr}^{2}+\mathrm{pa}(4) \cdot \mathrm{ncorr}+\mathrm{pa}(5)
$$

where $p a$ is a vector containing the polynomial coefficients from the polyfit function and $n c o r r$ is the corrected speed. The other parameters $b$ and $z$ are evaluated in the same manner. 
Given a known pressure ratio and speed we want to know the corresponding mass flow. In the original map this might be a problem since the curves for the lower speeds are not monotonic, i.e. there is no unique solution to the problem. With the use of elliptic curves we get monotonic yet accurate functions. An obvious drawback with the method is the large manual part of the function fitting. It makes the process less automatic, when new compressors are introduced, but on the other hand it gives a model that works for the whole operating range. The errors introduced come firstly from the curve fitting procedure, and secondly, new errors arise when the curves are parameterized with speed. The error of the mass flow model is around $2.9 \%$, around the normal operating point of $70000 \mathrm{rpm}$, see the result section, and is in general somewhat higher for lower speeds. Especially when the gas turbine operates near the surge line where the model curve is almost flat; a small error in the pressure ratio gives a larger error in the mass flow. However the gas turbine operates only in the lower regions during the start up or stop procedure and then the functionality of the model is emphasized over absolute accuracy.

The surge points and choke points for each speed are gathered and parameterized with speed. During simulation these polynomial functions are evaluated with the current speed to get an estimate of how close the current operating point is to the surge and choke line.

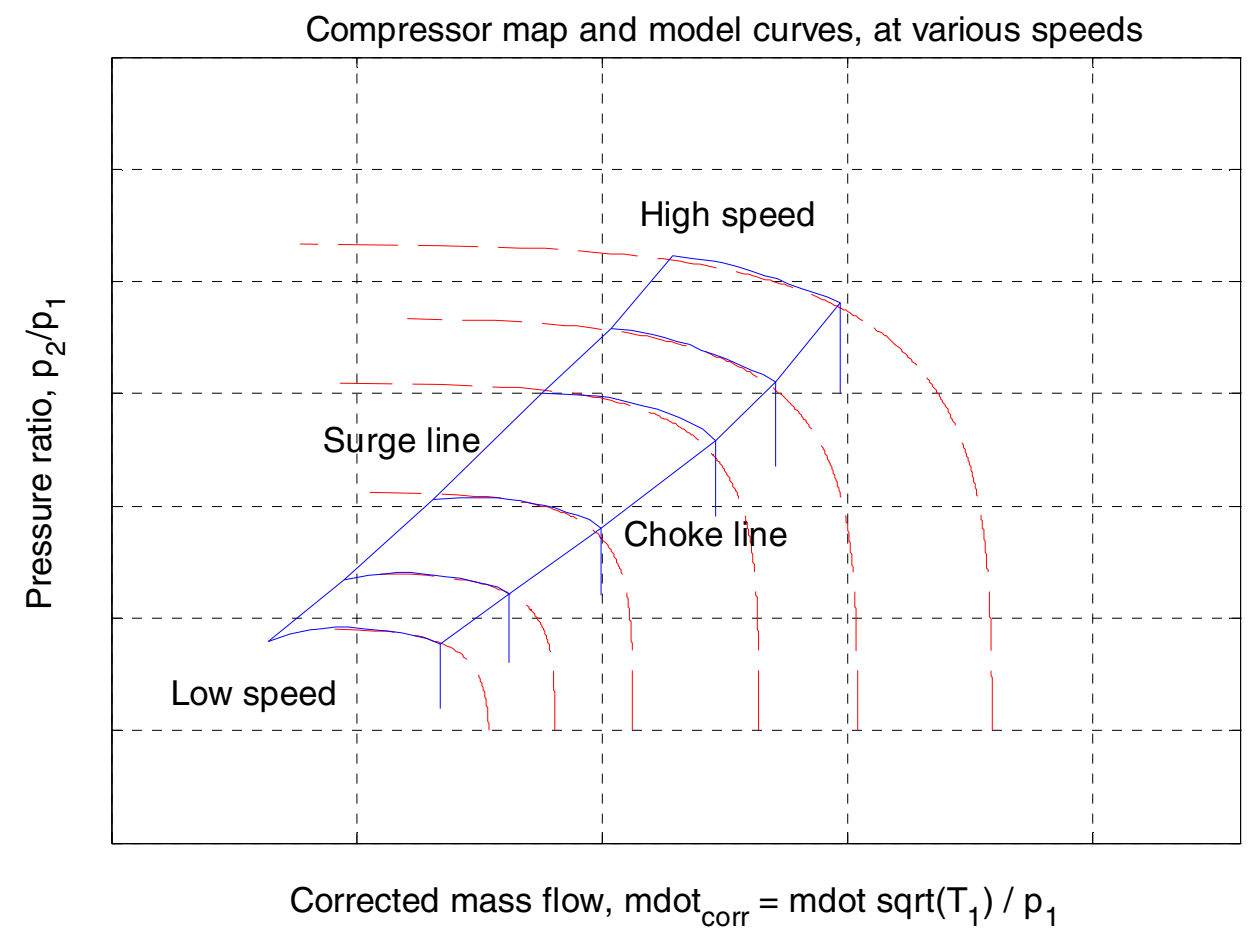

Figure 17: Compressor map (solid blue) and fitted ellipsoid curves (dashed red)

Summary of the development of the mass flow model of the compressor:

1. The value of the parameter $a$ is taken from the map, i.e. the mass flow value at choking conditions.

2. The parameter $z$ is set to an arbitrarily value, e.g. 5 .

3. One data point $(x, y)$ is taken from the map, where the ellipsoid curve and data will be identical matched. Using this point, equation (5.2.4) is solved for the parameter $b$.

4. The curve is plotted and compared to data from the map. By visual inspection, the parameters $a$ and $z$ are modified to ensure a better fit inside the operating range. 
5. For each speed there is a different set of parameters. The values of the parameters are now parameterized with speed in Matlab to polynomial functions to achieve a continuous model.

6. With the complete model, the mass flow is now continuously given by speed and pressure ratio.

Modelling the isentropic efficiency was harder. As can be seen in the figure below, there is no ellipsoid form to use. For a given rotational speed, the maximum efficiency is well-known, i.e. the top value of the curve. Knowing the maximum efficiency, we can approximate the efficiency map with parabolic degradation curves. The amount of curvature or degradation is denoted $d$ and is fitted based on numerous data to get parameterization for all speeds. The following equation is taken from Gustafsson (1998).

$$
\eta=\eta_{\max }-d\left(\dot{m}-\dot{m}_{\max e f f}\right)^{2}
$$

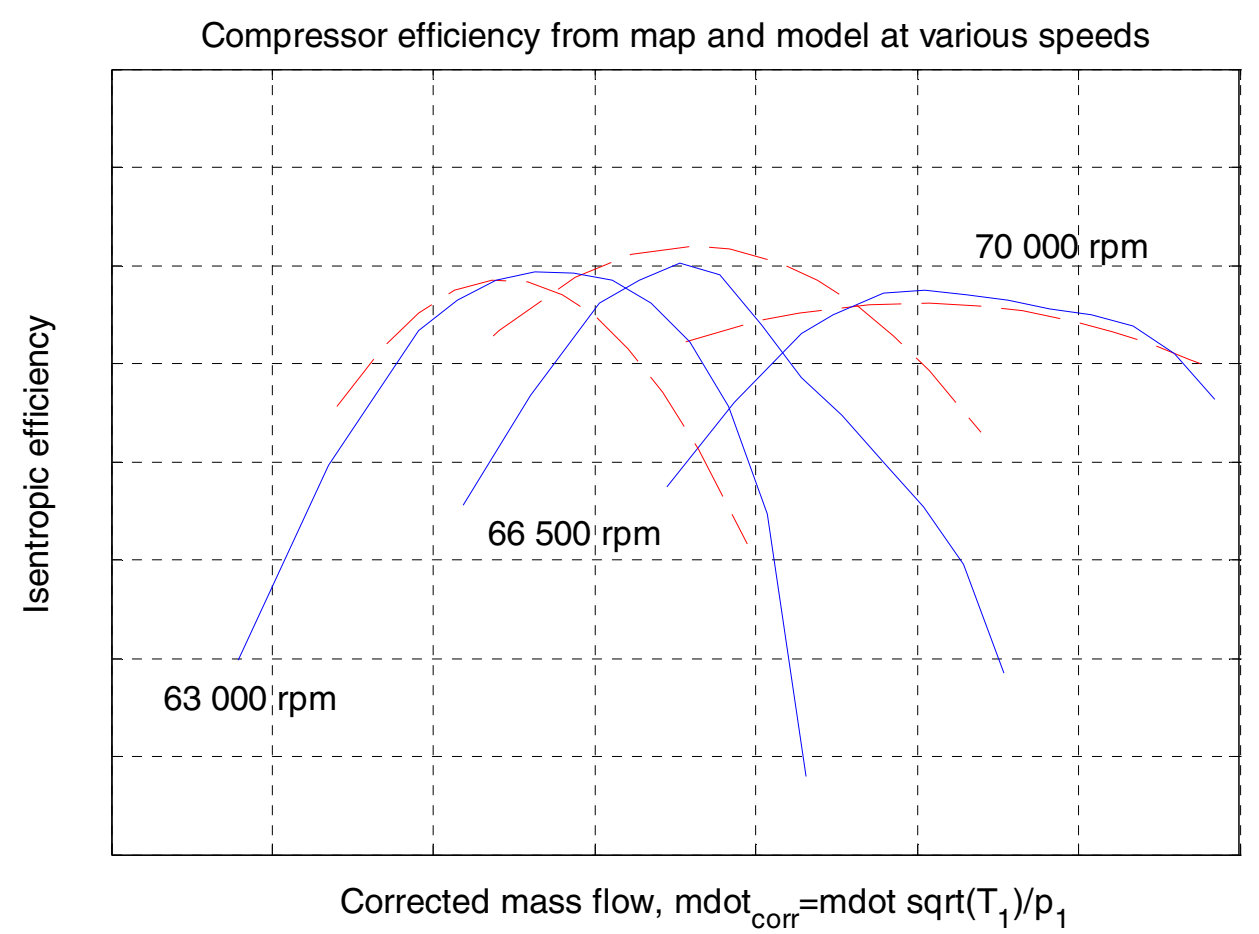

Figure 18: Isentropic efficiency from map (solid blue) and model (dashed red) as a function of speed and mass flow

As can be seen in the figure above, the curves are not exactly symmetrical. Near choking conditions (to the right), the performance is decreased rapidly. Another difficult part in this case is that the curvature changes a factor of 20 from the lowest to the highest speed. It is hard to create a continuous function that gives the correct value at every speed. Instead a simple interpolation function was used. At steady state the microturbine is very close to optimum, where the model is very accurate. At the extreme ends of the operating range, as e.g. near surge and choking, the accuracy decreases, due to errors both in the modelled mass flow and modelled efficiency.

So why did the interpolation method not work for the compressor mass flow or efficiency? The first obvious drawback is that for lower speeds the map does not have a unique solution for a given pressure and speed. As we did for the continuous ellipsoid curves we can manually change 
the pressure curves such that we have a monotonic map. A more important drawback is that the derivative of the mass flow would not be continuous with a bilinear interpolation. This can lead to numerical problems in the simulation. For some parameters like mass flow and pressure it is essential to have the derivative continuous, in order to do physically correct modelling. There are other interpolation methods, e.g. using cubic splines to generate continuous maps. The drawback here is then that we need to calculate 16 spline coefficients for each grid point in the interpolation map. In our case with 176 grid points and four interpolation tables we would need to calculate 11264 coefficients. Doing good modelling with interpolation methods is not an easy subject and is beyond the scope of this thesis.

The bilinear interpolation method was tested for the compressor mass flow and efficiency, but could not prove to be reliable in the whole operating range, even after the compressor map had been manually altered to provide unique solutions for lower speeds. In most cases the simulation was halted or got stuck due to chattering (discontinuity sticking). What happens is that a variable, e.g. speed is changed causing the interpolation index of the axis of the interpolation table to also change. The interpolated output data, e.g. the mass flow, causes the speed to change back and then the index is changed back as well and so on and so forth. This might be partly caused by the fact that the data is not rectangular. With this means that each speed has a different pressure ratio interval, e.g. for $20000 \mathrm{rpm}$ the pressure ratio ranges from 1.18 to 1.02 , whereas for $70000 \mathrm{rpm}$ the pressure ratio ranges from 5.2 to 4.8 . This means that e.g. index 1 (the first vertical column) represents different pressure ratios depending on speed. The turbine data, however, is in rectangular form. All the speeds have the same range of pressure ratio and e.g. the index 1 (the first vertical column) always represent data at a pressure ratio of 1.25 . The reasons behind this are simple. The compressor compresses the air and can for a certain speed only create a certain maximum pressure ratio. The higher the speed the higher is the pressure ratio created. The turbine expands the gas and work for all different pressure ratios and speeds, since it uses the pressure ratio created by the compressor. Another problem is when the numerical solver of the $\mathrm{n}$ :th order expects to find equations that are $\mathrm{n}$ times differentiable and the equations are only differentiable of a lower order. Often the solver succeeds despite this, but there are always reasons to be careful.

The mechanical efficiency of the compressor is taken to $100 \%$, since the major friction losses in the microturbine come from the two bearings, which are modelled in a separate submodel, see section 5.10.

\subsection{The turbine equations}

Before the exhaust gas enters the turbine, it passes through a nozzle where the velocity is increased and the pressure decreases. The high-speed gas exerts a force on the turbine blades and the geometry of the blade causes the turbine to rotate, thus producing mechanical work. After the turbine rotor, the gas passes through the diffuser of the turbine, where again the velocity is decreased and the pressure increased but not as much as after the compressor.

The thermodynamic equations are in large parts the same as for the compressor. Instead of repeating all of the derivations, there will be references to the corresponding compressor section.

We have the same energy balance as we used for the compressor. Also the same approximations can be made about the turbine. The only difference is that work is now done by the gas on the surroundings, i.e. on the turbine. The equation for the isentropic work of the turbine is therefore exactly as equation (5.1.9) but with the sign changed.

From equation (5.1.8) we get the relation for isentropic expansion and by including the isentropic efficiency from equation (5.1.10), the equation for the produced actual work can be rewritten as: 


$$
w=\eta_{i s}\left(\frac{\kappa}{\kappa-1}\right) R T_{1}\left(1-\left(\frac{p_{2}}{p_{1}}\right)^{\frac{\kappa-1}{\kappa}}\right)
$$

For increasing pressure ratios, an increase in isentropic efficiency of the turbine can be seen. A physical explanation is that the increase in temperature due to friction in one part of the expansion can be recovered as work on the turbine in the next part, Cohen (1996).

The produced power from the turbine is then:

$$
P_{\text {turbine }}=\dot{m} \cdot \eta_{i s}\left(\frac{\kappa}{\kappa-1}\right) R T_{1}\left(1-\left(\frac{p_{2}}{p_{1}}\right)^{\frac{\kappa-1}{\kappa}}\right)
$$

The turbine is mounted on the same shaft as the compressor, producing enough torque to power the compressor and the generator. Similar to the compressor, the turbine has a mechanical efficiency, depending on design and construction. The relation between the mechanical torque and the power produced can be written as:

$$
P_{\text {turbine }} \cdot \eta_{\text {mec }}=\tau_{\text {turbine }} \cdot \omega
$$

\subsection{The turbine model in Modelica}

The turbine is like the compressor modelled as a flow model, thus neglecting its small volume. The equations presented earlier describe the power produced by the turbine as a function of mass flow, the pressure ratio and temperature. To know what pressure and temperature we have at the equilibrium state, we need information about the correlation between the mass flow, rotational speed and pressure ratio. Like the compressor all this information is contained in a turbine map produced by Volvo Aero Corporation AB, see figure 19 below.
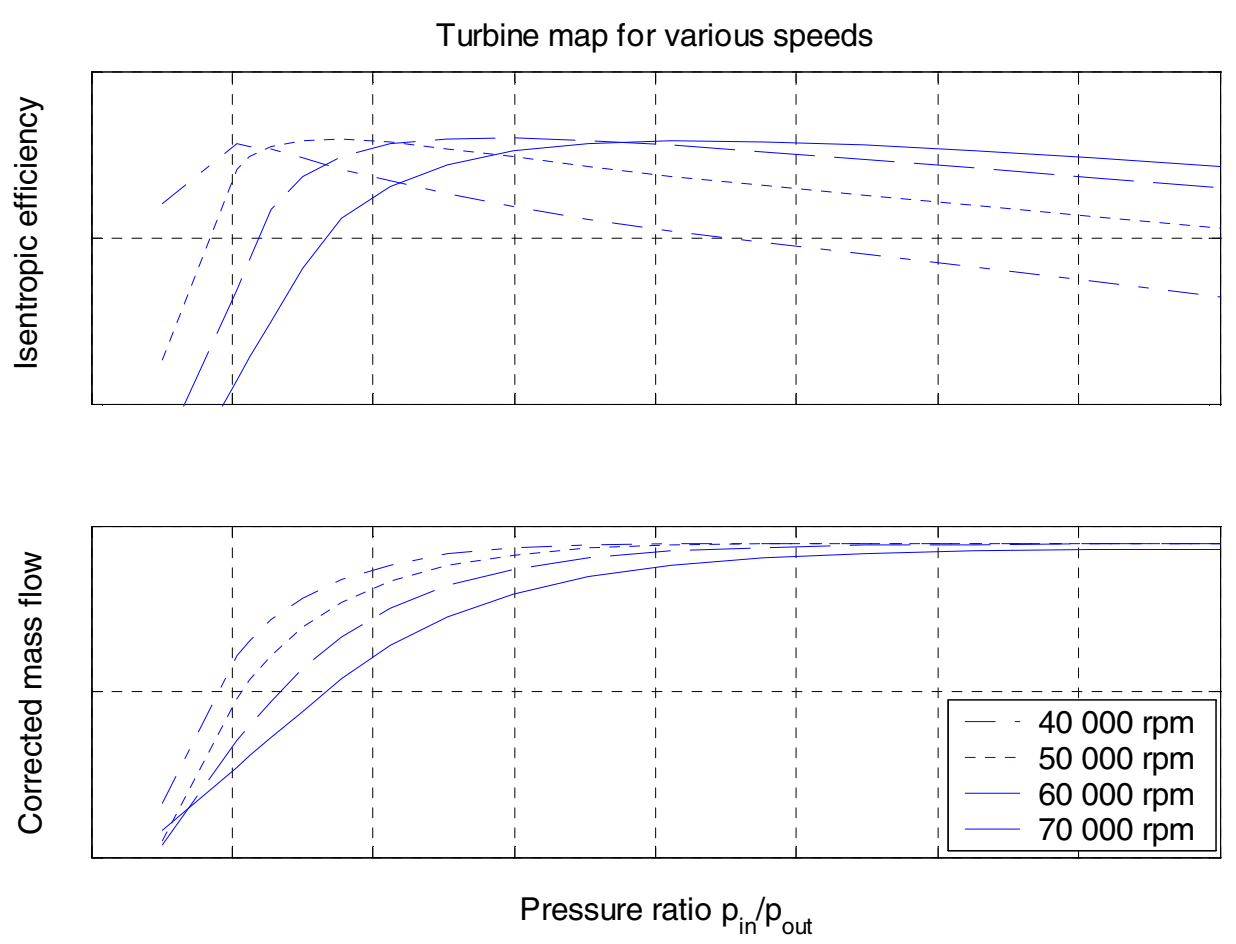

Figure 19: Turbine map generated by Volvo Aero Corporation AB 
In figure 19 we can see that the corrected mass flow, at large pressure ratio, has a constant maximum for all possible speeds. We can therefore assume the approximation that the turbine almost always works at choked conditions, i.e. the corrected mass flow is at or near maximum. With the approximation, we can use the following equation to calculate the constant maximum value of the corrected mass flow, Cengel (1998).

$$
\frac{\dot{m} \sqrt{T_{1}}}{p_{1}}=\frac{A_{t h r} M \sqrt{\kappa}}{\sqrt{R\left(1+\frac{\kappa-1}{2} M^{2}\right)^{\frac{\kappa+1}{\kappa-1}}}}
$$

The variable $A_{t h r}$ is the smallest nozzle throat area (the cross-section area) at the inlet to the turbine. The variable $M$ is the Mach number, defined as the ratio of flow speed and the speed of sound. The equation has its maximum at $M=1$, where the turbine is fully choked. CFD calculations of the turbine flow in the T100 at full load, carried out by Volvo Aero Corporation $\mathrm{AB}$, give a value of $M$ very close to 1 , which indicates that the approximation is justified. The figure 20 below shows the turbine mass flow model at a simulation of successive load changes. The model data is plotted and compared to the values the turbine map would have given the same conditions, calculated by interpolation. The model is closely matched to the computed values from the map, which can be seen in figure 20 below. The corrected mass flow model is approximated as a constant, even though the value actually increases some, due to an increase in $\kappa$. The map value shows a small decrease in corrected mass flow for lower part loads. The error at $50 \mathrm{~kW}$ load is however only $2.7 \%$.

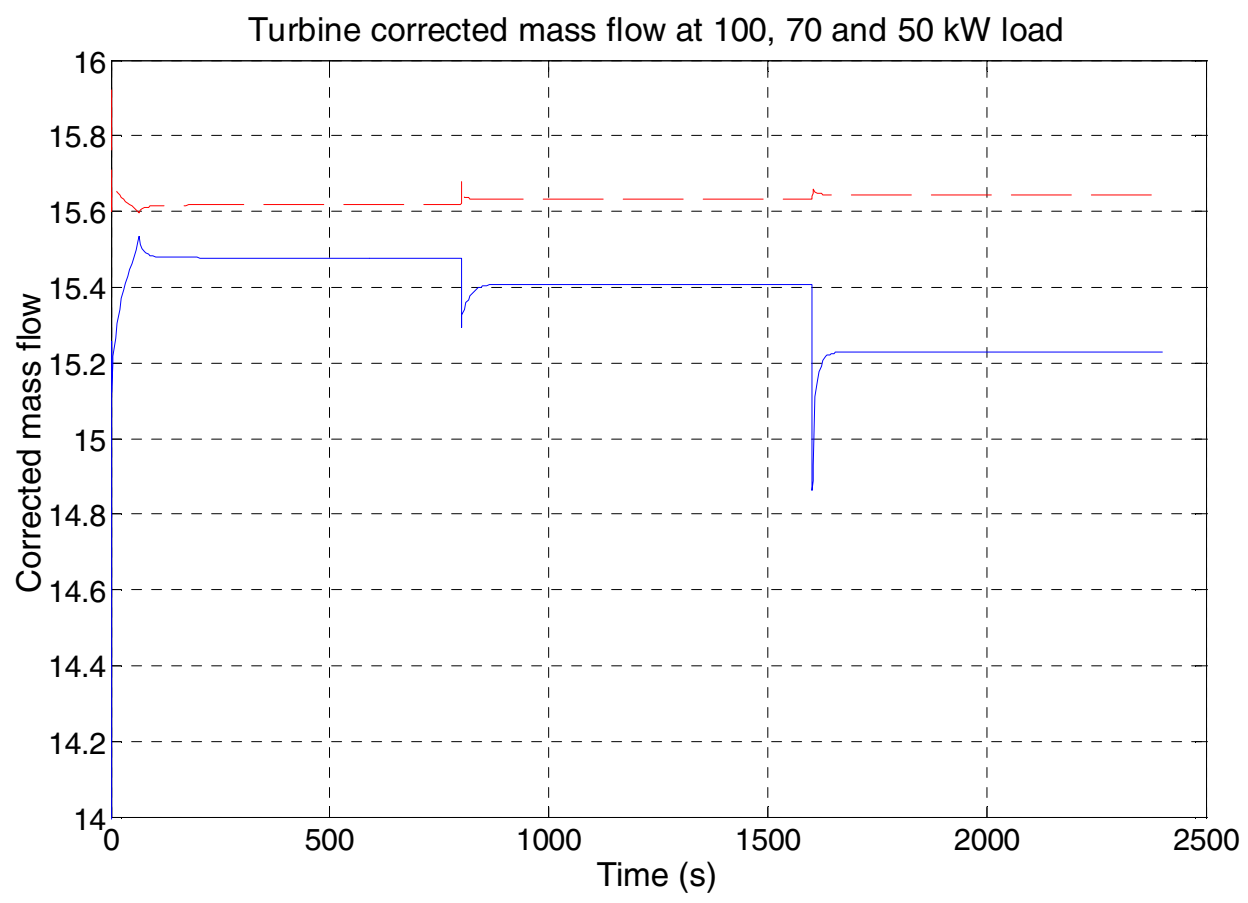

Figure 20: Turbine corrected mass flow model (dashed red) compared to turbine map (solid blue)

The efficiency data from the turbine map can be seen in figure 19. There is no obvious maximum or curve shape that can be used in the modelling. Instead a bilinear (2-dimensional) interpolation method was used. As have been explained in the compressor section, there are numerous problems with this method. However, it did work for the efficiency of the turbine. A reason for this is that the efficiency data is stored in rectangular form, see the compressor model section 
The axes of the turbine efficiency map consist of the speed and pressure ratio in monotonically increasing vectors, speed_vec and $p r$. Given a speed and a pressure ratio, the speed vector is searched to find the lowest index $i$ of the fitting speed interval. The procedure is repeated for the vector of the pressure ratio and the corresponding index $j$ is found. Now there are four possible efficiency values (the end points of the two fitting intervals) to interpolate between. These four values are the corners of the interpolated field.

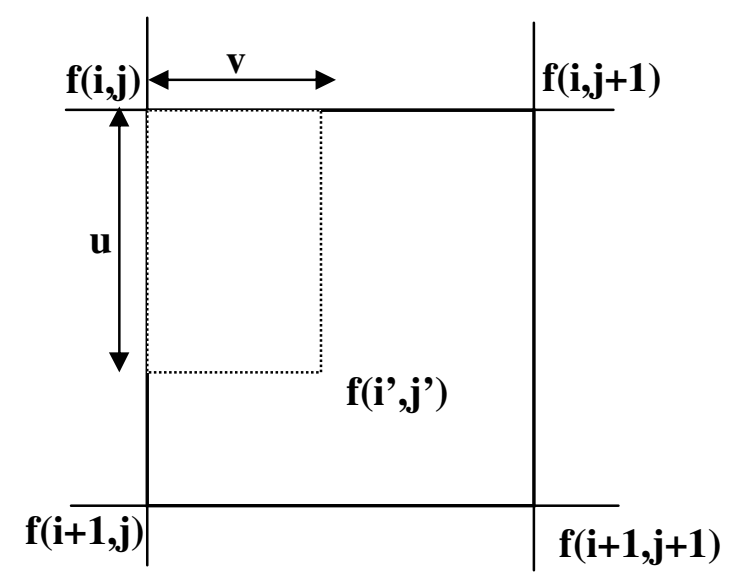

Figure 21: A point to be interpolated, surrounded by four grid points

The variables $u$ and $v$ are the normalized distances (between 0 and 1), according to the following equation:

$$
\begin{aligned}
& v=\frac{r-p r_{-} v e c[j]}{p r_{-} v e c[j+1]-p r_{-} v e c[j]} \\
& u=\frac{\text { speed }- \text { speed_vec }[i]}{\text { speed_vec }[i+1]-\text { speed_vec }[i]}
\end{aligned}
$$

where $r$ is the actual pressure ratio, speed is the actual speed and speed_vec and pr_vec are the axes of the interpolation table. The interpolation equation is then given by:

$$
f\left(i^{\prime}, j^{\prime}\right)=(1-u)(1-v) \cdot f(i, j)+(1-u) v \cdot f(i, j+1)+u(1-v) \cdot f(i+1, j)+u v \cdot f(i+1, j+1)
$$

With the interpolation method, no new errors are introduced and the accuracy of efficiency model depends solely on the accuracy of the turbine map.

The pressure ratio used as the input to the interpolation method is the pressure ratio excluding the turbine diffuser. The diffuser recovers some pressure and the velocity of the gas is decreased. The diffuser increases the efficiency of the turbine, since the turbine with a diffuser can produce the same amount of work but with a lower pressure ratio. In the turbine model, the diffuser is not included, but the efficiency of the turbine is increased by $1 \%$ to reflect the effect of the diffuser.

The mechanical efficiency of the turbine is taken to $100 \%$, since the major friction losses in the microturbine come from the two bearings, which are modelled in a separate Loss model, see section 5.10. 


\subsection{The heat exchanger}

The function of a heat exchanger is to transfer heat from one medium to another, often separated by a solid wall. It can be used in space heating, air-conditioning, power production and chemical processing. The following information and more can be found in DeWitt (1996) and Gustafsson (1998). There are different heat exchangers depending on flow arrangement and type of construction. The simplest heat exchanger is a concentric tube where the fluids flow in either parallel direction or in a counterflow manner. Another version is the crossflow heat exchanger where the fluids meet perpendicular to each other.

There are numerous methods of analysis, the two most common are the log-mean temperature difference and the effectiveness (efficiency) -NTU method. The log-mean method is used when the outlet temperatures are given by specifications or can be readily determined from the energy balances. It is also used in heat exchangers with low order of discretisation. In other cases the effectiveness-NTU method is to be preferred. The outlet temperatures are unknown in the T100 and therefore only the effectiveness-NTU method will be described.

The recuperator of the T100 is a counterflow heat exchanger, even though there is some crossflow in the beginning and in the end, see figure 7 . The hot fluid is the flue gas from the turbine and the cold fluid is the compressed air on the way to the combustion chamber. The heat exchange is modelled in the following way.

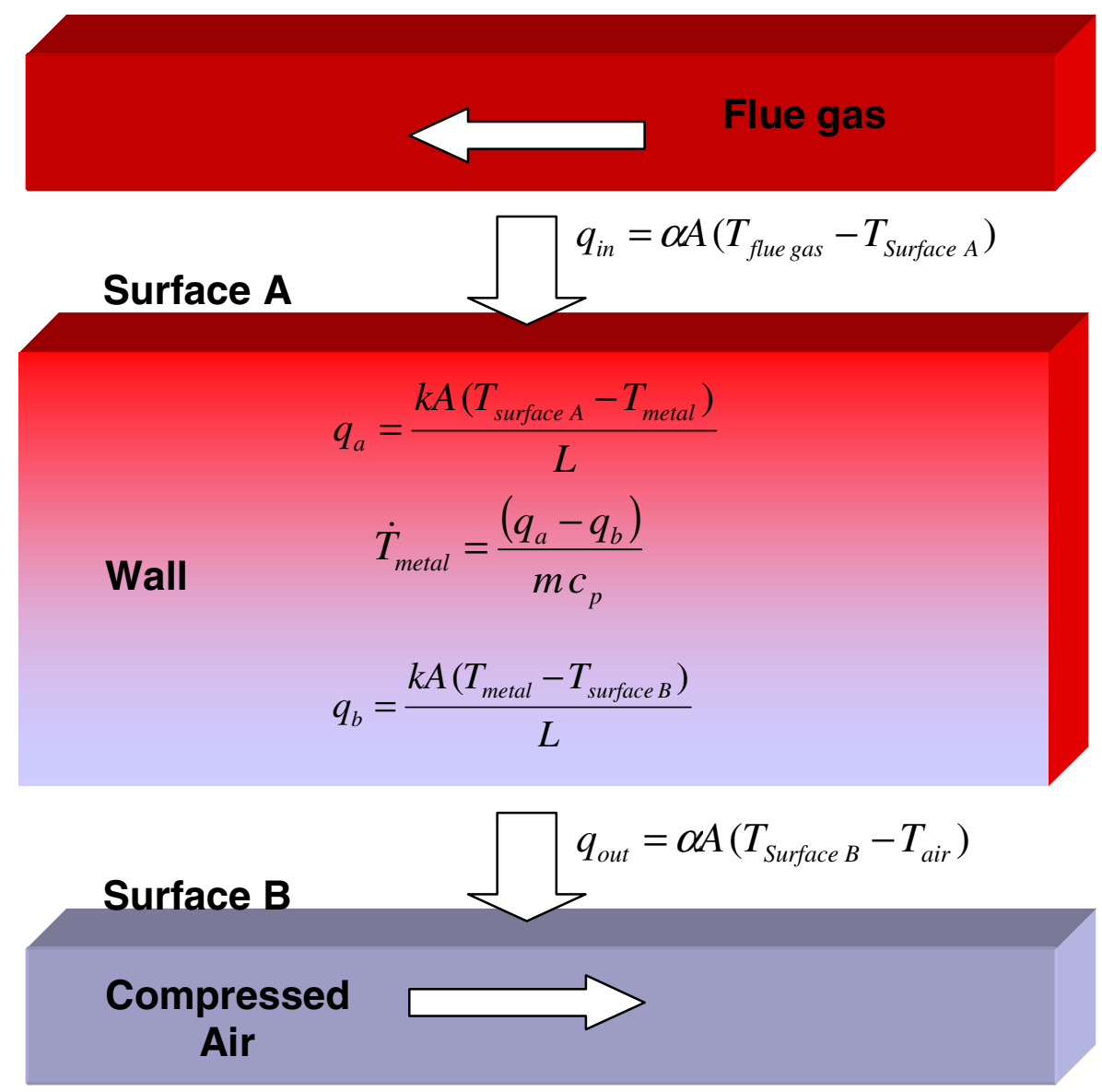

Figure 22: The heat equations for convection and conduction for 1-dim wall 
where $\alpha$ is the convection heat transfer coefficient, $k$ is the conduction heat transfer coefficient, $L$ is the thickness of the wall, $m$ is the mass of the wall and $c_{p}$ is the specific heat of the metal in the wall. These equations describe the dynamics of the temperature in the wall. The heat transfer is approximated to the 1-dimensional case, but since the wall is very thin compared to the length, it is a justified approximation.

We also want to define the efficiency of a heat exchanger, so that we know how good it is compared to an ideal heat exchanger. In order to do so we start by defining the maximum possible heat transfer rate:

where

$$
q=C_{\min }\left(T_{\text {flue gas, in }}-T_{\text {air }, \text { in }}\right)
$$

$$
C_{\text {min }}=\min \left\{\left(c_{p} \dot{m}\right)_{\text {air }},\left(c_{p} \dot{m}\right)_{\text {flue gas }}\right\}
$$

$\mathrm{C}_{\min }$ is minimum heat capacity flow of the two involved fluids. The subscripts in and out refer to the inlet and outlet of the heat exchanger and the subscript air and flue gas refer to the cold and hot fluid of the heat exchanger respectively. The maximum heat transfer can only be achieved in an ideal counterflow heat exchanger of infinite length. The actual heat transfer can thus be stated as:

$$
q=C_{\text {air }}\left(T_{\text {air }, \text { out }}-T_{\text {air }, \text { in }}\right)
$$

which gives us a formal definition on the efficiency:

$$
\mathcal{E}=\frac{q}{q_{\max }}=\frac{C_{\text {air }}\left(T_{\text {air }, \text { out }}-T_{\text {air }, \text { in }}\right)}{C_{\min }\left(T_{\text {flue gas, in }}-T_{\text {air }, \text { in }}\right)}=\frac{T_{\text {air }, \text { out }}-T_{\text {air }, \text { in }}}{T_{\text {flue gas, in }}-T_{\text {air }, \text { in }}}
$$

In the T100 microturbine, the fluids are air and flue gas. The only differences between the substances are somewhat lower percentage oxygen and higher percentage carbon dioxide and water vapor. When the fuel is added, the total mass flow is increased, but only with $1 \%$ due to the large air/fuel ratio. The exhaust gas has also higher temperature than the air, which also gives a little larger $c_{p}$. Then $C_{\min }=C_{\text {air }}$ and we get the right had side of equation (5.5.4).

The equation above gives the efficiency of the heat exchanger based on temperature measurements. It is used to evaluate the efficiency of a recuperator in use. It does not, however, say anything in advance about what the efficiency a designed recuperator will have and what factors that can improve the efficiency. Another useful expression for the efficiency is therefore taken from DeWitt (1996). The following equation is only valid when the heat capacity flows of the fluids are equal, which is a justified approximation in the case of the T100 microturbine.

$$
\varepsilon=\frac{N T U}{1+N T U}
$$

The variable $N T U$ (number of thermal units) is a dimensionless parameter that is widely used for heat exchangers. It shows how well the heat exchanger can transfer heat due to its geometry, mass flow, mediums and heat transfer coefficients.

$$
N T U=\frac{U \cdot A}{C_{\min }}
$$

where $A$ is the heat exchanger surface area and $U\left[\mathrm{~W} / \mathrm{m}^{2} \mathrm{~K}\right]$ is the overall heat transfer coefficient defined as

$$
U=\frac{1}{\left(\frac{1}{\alpha_{\text {air }}}+\frac{L}{k_{\text {wall }}}+\frac{1}{\alpha_{\text {fluegas }}}\right)}
$$

The variable $\alpha$ is the convection heat transfer coefficient; $L$ is the thickness of the wall and $k$ is the thermal conductivity (conduction heat transfer coefficient). The wall between the fluids is 
very thin so the main obstacle for the heat flow will be the convection from wall to fluid and not from one edge of the wall to the other edge. This means that the middle term of equation (5.5.7) can be neglected. This does not mean however that the wall is of no importance. The mass of the wall is very important for dynamic reasons, since heat will be stored in the wall.

As a summary, we can see that important factors for the efficiency of a heat exchanger are the surface area A, the convection heat transfer coefficient $\alpha$, the mass flow and the specific heat capacity of the mediums.

\subsection{Pipes and pressure loss models}

All pipes in the model consist of a control volume and a flow model. The flow characteristics such as pressure drop are included in the flow model. There are many variables concerning the pressure loss for a simple pipe such as friction, bends, inlets, outlets and height differences. It is a task beyond the scope of this thesis to do a detailed pressure loss model of a complicated machine like a gas turbine. In Gustafsson (1998), detailed pressure loss equations for a recuperator are derived. In the end Gustafsson needed to do manual adjustments of the temperature measurements to calibrate the model to experiment data. In the equations there are also some theoretical values that need to be estimated. Another source, Thomas (1999), was chosen to get pressure loss equations for a normal pipe.

$$
d p=\frac{\dot{m}^{2}}{\rho} C
$$

where $C$ is a constant factor due to friction, bends and inlets/outlets. The constant $C$ can be measured or estimated in a certain design point. Then we can choose a standard pressure loss model from the ThermoFluid library and modify it to deal with large thermal changes.

$$
d p=\frac{\dot{m}^{2}}{\dot{m}_{0}^{2}} \frac{\rho_{0}}{\rho} d p_{0}
$$

where $\mathrm{dp}_{0}, \operatorname{mdot}_{0}$ and $\rho_{0}$ are constant pressure loss, mass flow and density at a known design point and together they form the constant factor $C$. This is an easy-to-use formula that gives reasonable results. Since my thesis emphasizes the model of the entire system, there is no time to create and tune a more detailed pressure loss as in Gustafsson (1998).

\subsection{The heat exchanger model}

The two heat exchangers in the T100 microturbine, the recuperator and the gas/water heat exchanger are both modelled in the same way, except for the medium models used.

In the ThermoFluid library, there are complete pipe models with heat conduction and with air or flue gas as medium. The pipe model inherits code for each important property, as e.g. pressure drop, geometry and medium, see figure 12 . The heat exchangers are then modelled as two parallel pipes with a wall in between. There is no difference in the model for counterflow or parallel flow, since the direction of the flow is decided by the pressure, which is originally set by the user. The wall is modelled as a simple dynamic wall with a homogenous temperature throughout the wall. It inherits code from classes as e.g. geometry and heat conduction. The pipes and the wall are then connected with heat connectors so that the heat equations of each component are linked together. 


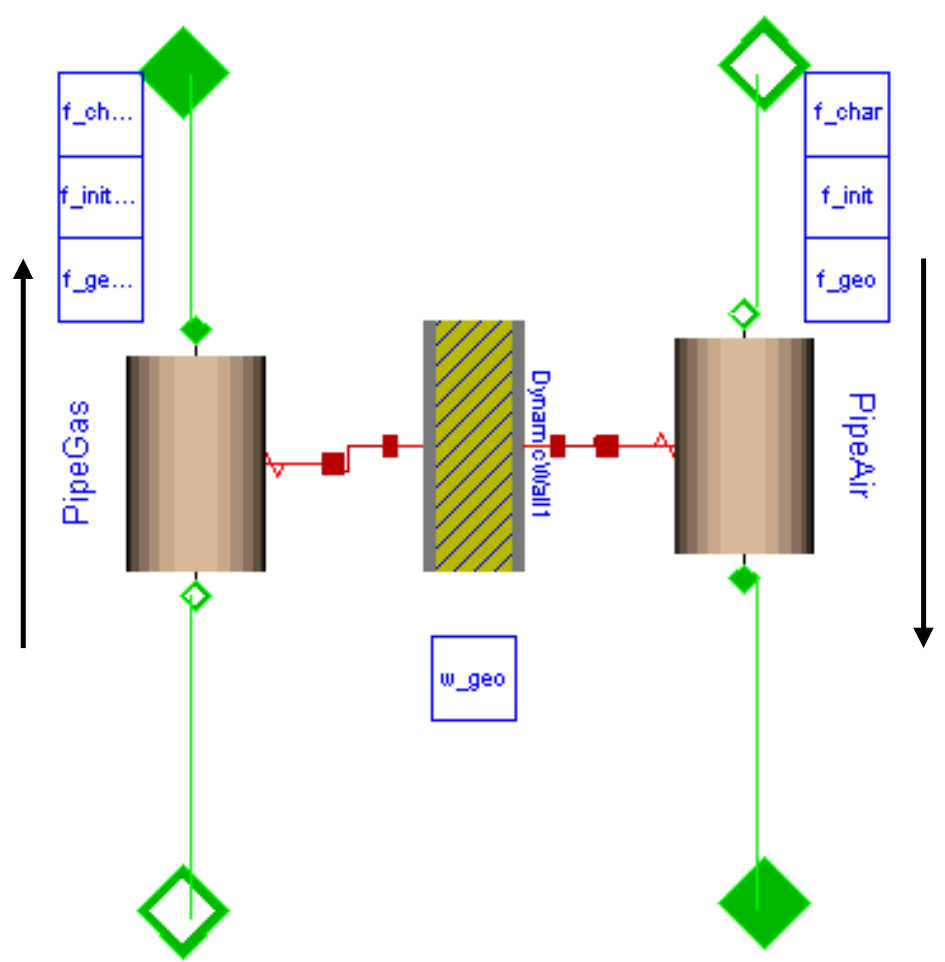

Figure 23: The heat exchanger model with arrows indicating counter flow pattern

The heat exchanger can be discretisised by the user via a parameter. At discretisation the control volume and the flow model of the pipe are discretisised, so that the control volumes and flow models come in alternating sequence. A higher degree of discretisation gives a higher accuracy in the model. On the other hand, smaller discretisation volumes give smaller time constants and stiffer model as well as increasing the number of dynamic states. The computation time increases with the stiffness and the number of states, but also depends on the transients to be simulated.

The model error, due to discretisation, can be seen in figure 24 , below. The heat exchanger is divided into $\mathrm{n}$ separate smaller control volumes and flow models. In each control volume the temperature is, as previously mentioned, an average temperature of that control volume. If we want to know the outlet temperatures of the heat exchanger, it is the temperature of the last control volume in the flow direction that will be used. That temperature, however, is still only the average temperature and not the exact outlet temperature. The model error decreases for a higher order of discretisation but again, there is a trade off between accuracy and computation time. Note also that the model error is larger for the side of the heat exchanger, where the cold air exits. It is in that part, where the temperature curve increases the most. On the opposite side, where the hot gas exits, the model error is much smaller, since the temperature curve is less steep. 


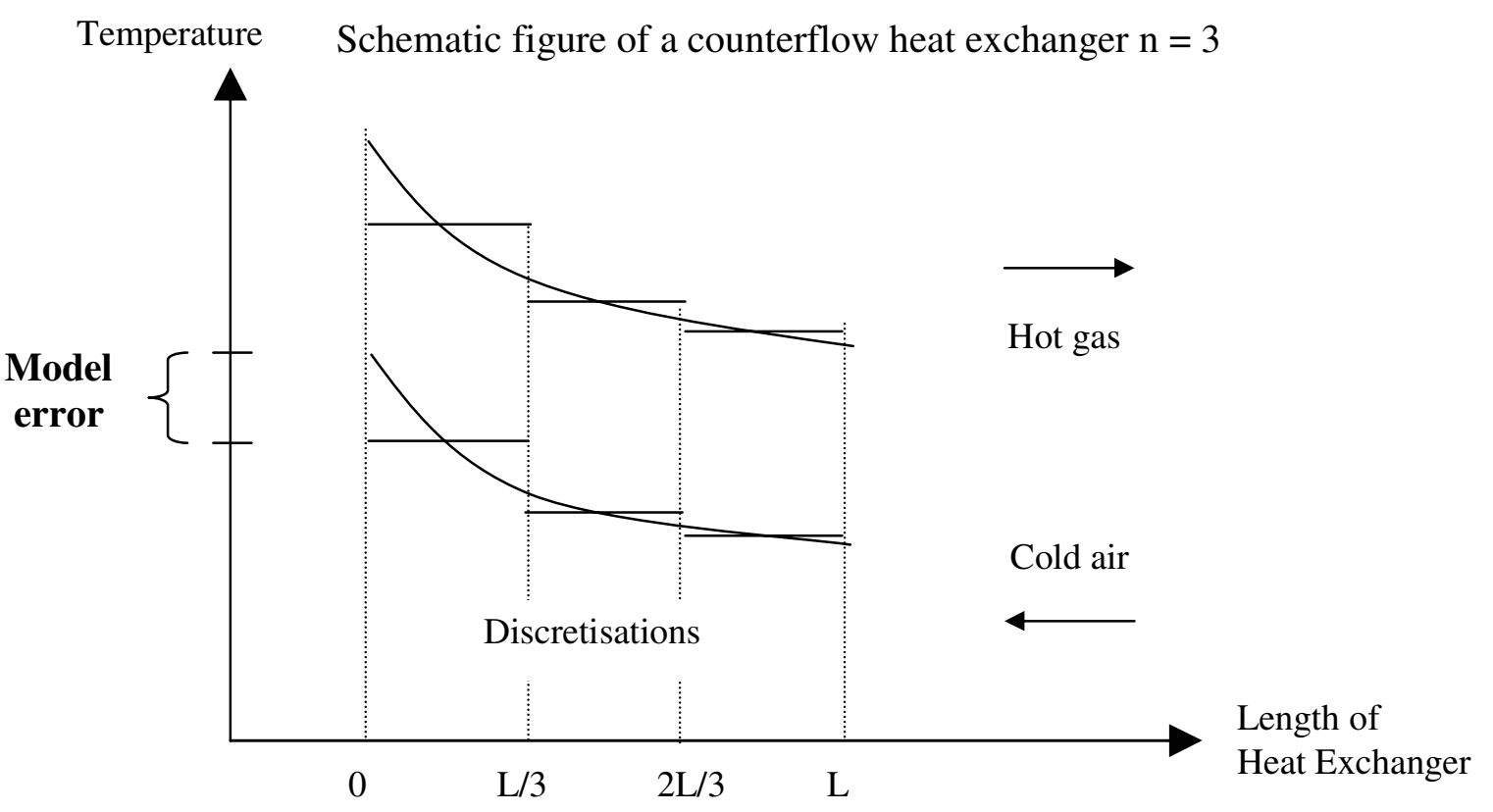

Figure 24: A schematic sketch of a counterflow heat exchanger with $\mathbf{n}=\mathbf{3}$

A third heat exchanger model is used to model the heat storage and transfer that occurs in the turbine rotor and turbine diffuser, as described in section 3.2. By adjusting the parameters of the extra heat exchanger model, the complete microturbine model received similar dynamics from TIT to TOT as can be seen in the real microturbine, see the dynamic verification section. Since almost all heat exchange is between the flue gas and the metal of the turbine and just a very small part of this continues to the compressed air, the heat convection coefficient is high on the flue gas side and very small on the air side. Therefore the heat exchanger has no static heat transfer at steady state, but only a dynamic effect at sudden load changes.

The values of the different parameters in the heat exchanger models are taken from the real machine, but some of them, e.g. the geometry and the convective heat transfer coefficient $\alpha$ need to be adjusted before being used in the simulation model. Even though the geometry of the heat exchanger model does not exactly look like the real recuperator, the volume and heat loss area are the same. With a given volume, a reasonable diameter and length is used. To compensate for model errors due to discretisations effects and the fact that there are cross flows instead of counter flows at the ends of the recuperator, the constant value of the heat transfer coefficient is manually adjusted. The value is adjusted so that the performance of the heat exchanger model fits the steady state data of the real recuperator.

\subsection{The combustion chamber}

In this section I will describe the theory and equations of the combustion chamber. For more theory on this topic, see Cengel (1998). The model of the combustion chamber is taken from Perez (2001), where a complete set of equations for all components in natural gas can be found. For simplicity, only equations for one component of natural gas, methane, will be derived. The equations for the other hydrocarbon fuels are similar, but with different numbers.

The combustion chamber is a reacting system compared to the compressor and other components, which were non-reacting. We therefore need to use the notion of chemical internal energy, which is associated with the destruction and formation of chemical bonds between atoms. In a gas 
turbine, the air is mixed with fuel and burned at constant pressure. The chemical bonds of the fuel atoms are destroyed and released energy heats up the exhaust gases. The default fuel in the microturbine is natural gas, even though the microturbine can be modified to run on swamp gas, kerosene, propane and other fuels. Natural gas does not consist of one substance. It is a mixture, where the main components are four different hydrocarbon fuels and nitrogen and carbon dioxide. The exact mass percentage varies for each mixture, but a common type of natural gas can have the following composition:

\begin{tabular}{|l|l|l|}
\hline Methane & $\mathrm{CH}_{4}$ & $81 \%$ \\
\hline Ethane & $\mathrm{C}_{2} \mathrm{H}_{6}$ & $7.9 \%$ \\
\hline Propane & $\mathrm{C}_{3} \mathrm{H}_{8}$ & $4.2 \%$ \\
\hline N-Butane & $\mathrm{C}_{4} \mathrm{H}_{10}$ & $4.7 \%$ \\
\hline Nitrogen & $\mathrm{N}_{2}$ & $1 \%$ \\
\hline Carbon dioxide & $\mathrm{CO}_{2}$ & $1.2 \%$ \\
\hline
\end{tabular}

Table 1: Mass percentage of a common natural gas mixture

To get the right chemical mixture after combustion, stoichiometric reaction formulas are used. We assume that the existing nitrogen and the carbon dioxide in the air and in the fuel as well as the water in the air are all inert gases, i.e. they do not react with anything $\left(\right.$ no $\left.\mathrm{NO}_{\mathrm{x}}\right)$ or contribute to the chemical reactions. We also assume complete combustion with an abundance of air, i.e. there are no remains of the fuel in the products. The stoichiometric relationship gives:

$$
\mathrm{CH}_{4}+2 \mathrm{O}_{2} \rightarrow \mathrm{CO}_{2}+2 \mathrm{H}_{2} \mathrm{O}
$$

The formula above shows the relationship in molar quantities. To get a mass flow balance we want to change the units. To convert the equation above to mass flow units we use the substances' molar mass M, i.e. the mass for one mole. Then we can rewrite the equation to:

$$
\mathrm{CH}_{4}+2 \cdot\left(\frac{M_{\mathrm{O}_{2}}}{M_{\mathrm{CH}_{4}}}\right) \mathrm{O}_{2} \rightarrow\left(\frac{M_{\mathrm{CO}_{2}}}{M_{\mathrm{CH}_{4}}}\right) \mathrm{CO}_{2}+2 \cdot\left(\frac{M_{\mathrm{H}_{2} \mathrm{O}}}{M_{\mathrm{CH}_{4}}}\right) \mathrm{H}_{2} \mathrm{O}
$$

The combustion occurs with an abundance of air, i.e. more air than needed according to the stoichiometric equations. This results in the existence of air in the products as well as in the reactants. Now we can put up equations for the mass flows of the different components in the flue gas leaving the combustion chamber.

$$
\begin{gathered}
\dot{m}_{\mathrm{CO}_{2}, \text { out }}=\dot{m}_{\mathrm{CO}_{2}, \text { in }}+\left(\frac{M_{\mathrm{CO}_{2}}}{M_{\mathrm{CH}_{4}}}\right) \cdot \dot{m}_{f u e l, C H_{4}}+\dot{m}_{f u e l, C O_{2}} \\
\dot{m}_{\mathrm{H}_{2} \mathrm{O}, \text { out }}=\dot{m}_{\mathrm{H}_{2} \mathrm{O}, \text { in }}+2 \cdot\left(\frac{M_{\mathrm{H}_{2} \mathrm{O}}}{M_{\mathrm{CH}_{4}}}\right) \cdot \dot{m}_{f u e l, C H_{4}} \\
\dot{m}_{\mathrm{O}_{2}, \text { out }}=\dot{m}_{\mathrm{O}_{2} \text {, in }}-2 \cdot\left(\frac{M_{\mathrm{O}_{2}}}{M_{\mathrm{CH}_{4}}}\right) \cdot \dot{m}_{f u e l, C H_{4}} \\
\dot{m}_{\mathrm{N}_{2}, \text { out }}=\dot{m}_{\mathrm{N}_{2}, \text { in }}+\dot{m}_{\mathrm{N}_{2}, \text { fuel }}
\end{gathered}
$$

The total mass balance equation is then:

$$
\dot{m}_{\text {out }}=\dot{m}_{\text {in }}+\dot{m}_{\text {fuel }}
$$

If the other hydrocarbon fuels are used, their individual contributions are added to the mass flow equations of the products. 
Before the energy balance is described, we first introduce the notion of enthalpy of formation $\mathrm{h}_{\mathrm{f}}$. It can be explained as the enthalpy of a substance at a specified state due to its chemical composition. A reference state is used, $\mathrm{T}=298.15 \mathrm{~K}$ and $\mathrm{p}=1 \mathrm{~atm}$. At this state, all stable elements have a value of $h_{f}=0$ and with a stable element we mean chemical stable elements like $\mathrm{N}_{2}, \mathrm{H}_{2}$ and $\mathrm{O}_{2}$ in their naturally occurring form, not the atomic versions of $\mathrm{N}, \mathrm{H}$ and $\mathrm{O}$. Using this reference we state:

The enthalpy of formation of a component, at the reference state, is the value of energy released or absorbed, when it is created from its primary stable forms $\left(\mathrm{O}_{2}\right.$ etc $)$ at the same reference state, Cengel (1998). A negative enthalpy of formation means that energy is released during the creation of the component and vice versa. An example is liquid propane, that has an enthalpy of formation of -118 $910 \mathrm{~kJ} / \mathrm{kmol}$. For each $\mathrm{kmol}$ of propane that is burned and reacts to carbon dioxide and water, $118910 \mathrm{~kJ}$ of energy is released. If the products or reactants are not in the reference state, the enthalpy of formation will vary. We introduce the notion of total enthalpy, which is the sum of enthalpy of formation at the reference state and the normal (sensible) enthalpy at the current state relative to the enthalpy at the reference state:

$$
\text { Enthalpy }=h_{f}^{0}+\left(h-h^{0}\right)
$$

where the superscript 0 indicates the value at the reference state $(T=298.15 \mathrm{~K}$ and $\mathrm{p}=1 \mathrm{~atm})$. Now we can state the energy balance equation from Cengel (1998).

$$
\dot{Q}+\dot{W}+d P E+d K E=\sum \dot{m}_{p}\left(h_{f}^{0}+\left(h-h^{0}\right)\right)_{p}-\sum \dot{m}_{r}\left(h_{f}^{0}+\left(h-h^{0}\right)\right)_{r}
$$

where the subscripts $p$ and $r$ denotes products and reactants. The changes in kinetic energy in the combustion chamber can be neglected together with the changes in potential energy. We also know that the volume is constant, which gives zero work done. The released energy is then used to heat the products of the combustion. The combustion chamber is well insulated but still has some heat losses. The heat losses and a possible incomplete combustion can be modelled with an efficiency of combustion, $\eta_{\mathrm{cc}}$. The energy balance will then be:

$$
\sum \dot{m}_{p}\left(h_{f}^{0}+\left(h-h^{0}\right)\right)_{p}=\eta_{c c} \cdot \sum \dot{m}_{r}\left(h_{f}^{0}+\left(h-h^{0}\right)\right)_{r}
$$

\subsection{The temperature sensor}

The temperatures in the model are given by evaluations in the control volumes. If we want to know the exact temperature at some other locations, e.g. after the compressor (a flow mods! $\$_{2}$ 1 1 ) need an independent temperature sensor. As mentioned earlier the temperature in the outlet flow connector of the flow model is the following control volume's temperature, which can be very different. Instead we can use the flow variables energy flow $\mathrm{q}_{\text {conv }}$ and the mass flow $\dot{m}$ to get the correct enthalpy:

$$
h(T)=\frac{q_{\text {conv }}}{\dot{m}}
$$

Using equation (4.4.1), which gives the enthalpy as a function of temperature, we can state an equation for Dymola to solve for temperature:

$$
R\left(-a_{1} T^{-1}+a_{2} \ln T+a_{3} T+a_{4} \frac{T^{2}}{2}+a_{5} \frac{T^{3}}{3}+a_{6} \frac{T^{4}}{4}+a_{7} \frac{T^{5}}{5}+b_{1}\right)-\frac{q_{\text {conv }}}{\dot{m}}=0
$$

Dymola solves the high-order non-linear equation and we get the correct temperature. The computations are fast and do not increase the simulation time significantly. In some cases as e.g. in the heat exchanger the true temperature does not differ much from the temperature in the following control volume, since the temperature drop depends on the pressure loss over the flow model, which is usually small. In other cases as e.g. right before the finishing gas reservoir the 
temperature is taken from the reservoir and the temperature in the flow connector can then be arbitrarily inaccurate.

The dynamics in the real temperature sensors are modelled as a first order filter with a time constant of 1.5 seconds and a unit gain.

\subsection{The Generator, Friction losses and Auxiliary equipment models}

The microturbine model in this thesis is focused on the thermodynamic and mechanical properties of the T100 microturbine. Therefore the model includes the mechanical work the generator consumes but not the electricity it generates. The electric power the generator is supposed to produce is given by the user via a parameter. The losses in the power electronics, which includes the electrical part of the generator, are modelled with an efficiency variable. Friction in the two bearings that supports the single rotating shaft, are modelled as a linear function of the electrical power generated. The friction model is taken from the DSA model.

The microturbine has auxiliary equipment that runs on its own generated electricity, thus reducing the amount of electrical power that can be supplied to the grid. The auxiliary equipment is the fuel booster, ventilation fan, oil pump, water cooling pump, oil separator and the buffer air pump.

Altogether the losses are gathered in a separate model that calculates the necessary engine power output, as a function of the desired electrical power output. The engine power output is defined as the difference in produced power from the turbine and the consumed power by the compressor.

$$
P_{\text {engine }}=\left(m+k \cdot P_{\text {electric }}\right)+\frac{1}{\eta_{p e}} \cdot\left(P_{\text {auxialiary }}+P_{\text {electric }}\right)
$$

where $m$ and $k$ are coefficients in the linear friction model and $\eta_{\text {pe }}$ is the efficiency of the power electronics. The engine power output and its corresponding torque are related to the total torque equation as follows:

$$
\tau_{\text {turbine }}+\tau_{\text {compressor }}+\tau_{\text {output }}=\frac{P_{\text {turb }}+P_{\text {comp }}+P_{\text {engine output }}}{\omega}=0
$$

where the turbine power and torque have negative values to reflect the producing capacity of the turbine.

\subsection{Flow Sources and Reservoirs}

The air flowing into the compressor is taken from the outside surroundings. In the model, the air flow is modelled as coming from an infinite source with a mass flow, temperature and pressure set by the user. A source is a control volume of infinite size, i.e. the states of the air are not changed regardless of how much air that is extracted from it. There are three possible inputs to the flow source, which can be used to vary the temperature, pressure and mass flow during simulation. The input signals act as the derivative of the properties, in order to maintain flow properties that are differentiable. In the source, the initial chemical composition is set if the medium has more than one component, e.g. air or natural gas.

The exhaust gases of the microturbine are rejected into the atmosphere, which is modelled as an infinite reservoir. A reservoir is the same as a source; the only difference is that mass is rejected to a reservoir and mass is extracted from a source. Since no mass is extracted from the reservoir, the properties mass flow and temperature have no significance in this case. Very important properties, however, are the pressures at the source and the reservoir, since it is the pressure difference that partially states the pressures in each component. The pressure difference is also important for the calculation of the mass flow. 


\subsection{The Control System}

In this section I will describe the general idea of the control system for each of the two modes, normal parallel mode and stand-alone mode, see section 3.2 for a description of these modes.

The main input to the regulator is an electric power reference, i.e. the amount of electric power the generator should produce. The main output of the regulator, is the fuel rate, i.e. how much fuel that will be injected in the combustion chamber. There is a maximum value, with the fuel valves fully opened and a minimum value, when the valves are open just do the flame is kept alive. The measurements that are used in the feedback loop are the speed, the power currently produced in the generator and the temperature after the turbine.

In the parallel mode, the gas turbine produce heat and power at a constant level to a local heating system and a power grid, which is connected to the national power grid. If the power demand in the local power grid increases to a higher value than the maximum output of microturbine, the extra power is taken from the outer power grid. The microturbine usually runs at its optimum, full load. Since the control system does not need to meet any sudden changes, all changes can be done slowly and the thermal fluctuations inside the gas turbine are minimized. The life span of a gas turbine is closely related to how large and fast temperature changes it has to experience, e.g. at step changes and start and stop procedures. The changes are made slowly by including a rate limit function. A rate limit model has been implemented, but it only works if the signal source is differentiable, e.g. a ramp or sinusoidal. The total efficiency is optimal at a certain temperature and speed, which makes the microturbine more economical if always run at the optimum point. The control system tries therefore to produce the demanded power and at the same time keeping the temperature and speed constant at the optimal value.

In the stand-alone mode the objective of the control system is to maintain a constant voltage and frequency of the local power grid regardless of the actual power load. This objective is much stricter than for the parallel mode and therefore the control system needs to be a lot faster. The faster control is achieved on the expense of higher thermal variations in the machine, thus reducing its life span. However this is a price one has to pay to achieve the objective of standalone power delivery. The control system consist of a normal speed regulator that tries to maintain a predefined reference speed, e.g. $63000 \mathrm{rpm}$. Even though that speed might not be the optimal for lower power loads it is necessary to have a speed buffer if the load is sudden increased, since it is hard to increase the speed without reducing the power load for a short moment. The regulator tries to maintain the speed by controlling the fuel input to the turbine. The upper performance limit here is the limitation of the fuel injectors. There is a maximum amount of fuel that can be injected and that is based on temperature limitations of the material before and after the turbine.

\section{Simulations and Verification}

As a part of the verification process a large number of simulations were made and some of them will be presented in this chapter.

\subsection{Simulations}

With a complete model it is open for the user to simulate the process under any circumstances the user chooses. There are numerous parameters that must be set for each simulation case. All of the parameters have a default setting to avoid tedious work and numerical problems in case the parameter is not correctly set. Default settings can however be very dangerous, since the user can simulate the model without having checked if the correct parameter values are used. Some 
important parameters that control the performance and equilibrium values of the microturbine will be described below.

With the choked mass flow model of the turbine, the mass flow and pressure ratio of the microturbine at equilibrium are determined by the throttle area parameter, $\mathrm{A}_{\mathrm{thr}}$. If the throttle area is increased, the equilibrium value of the mass flow will increase, while the corresponding pressure ratio value will decrease. From the blueprints of the microturbine we can get a initial estimation and then we have to adjust it so that the equilibrium values of the model corresponds to the measured equilibrium values of the real microturbine.

It is very important to have a correct value of the temperature of incoming air, since a gas turbine has a higher efficiency at lower air temperatures, see equation (2.4.1). The default air temperature is set to $18^{\circ} \mathrm{C}$ for the reason that the DSA data has been generated based on that temperature.

Other important parameters are the pressure of inlet air and flue gas at the outlet of the microturbine, i.e. the pressures in the source and the reservoir. The efficiency can be sensitive to small changes in pressure of the combustion chamber.

All simulations were solved by DASSL, a differential-algebraic-equations solver, in Dymola. The tolerance was set to a value of $1 \mathrm{e}-6$ for all simulations in this thesis. 


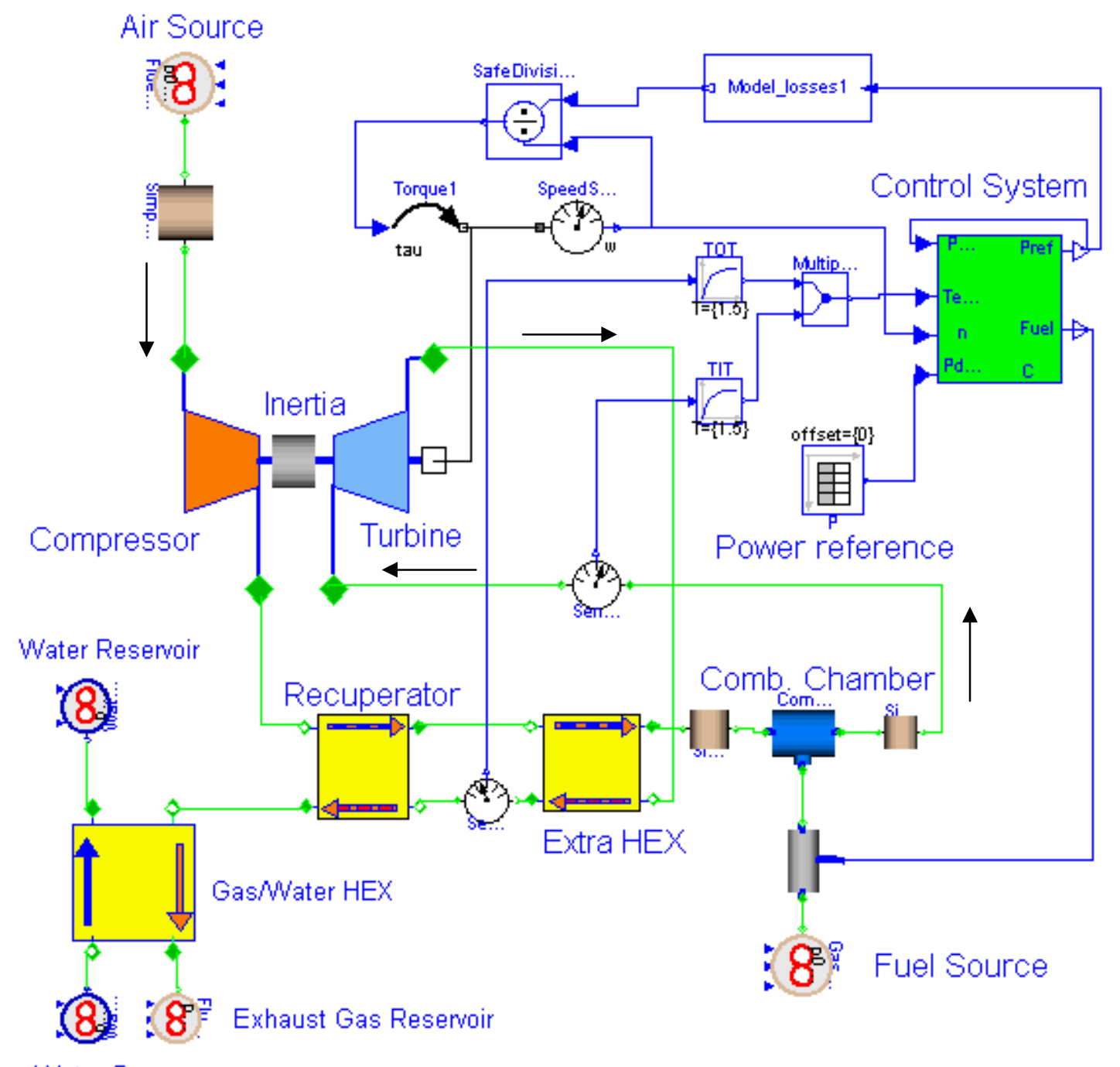

Water Source

Figure 25: The complete model

In the figure above, the complete model is presented. Green lines represent flows, like air, fuel and water. Blue lines represent signals to and from the control system. Extra arrows have been included to show the general direction of the gas flow.

Fresh air comes from the air source in the upper left corner, passes the compressor, and enters the recuperator. After the recuperator, there is an extra heat exchanger to model the heat storage in the turbine rotor and diffuser. The air continues into the combustion chamber, where it is mixed with the fuel and burned at a constant pressure. The exhaust gas enters the turbine, the extra heat exchanger, the recuperator and finally the gas/water heat exchanger. In the end, the exhaust gas exits the microturbine system into a reservoir.

The small white block on the right side of the turbine is the flange, to which the generator is connected. The generator does not have a separate model block, since it is only the mechanical work that the generator consumes that is modelled. The work and the corresponding torque are added on the single shaft via this flange. Between the compressor block and the turbine block, there is an inertia model of the single shaft. The circular blocks with hands are sensors. In the upper part of the figure a speed sensor can be seen, which delivers the speed measurement to the 
control system. The other two sensors connected to the green flow lines are temperature sensors, which are connected to a first order filter to model the dynamics in the sensors, before they are come to the control system block. In the upper right corner, there is a block for the model losses, the function that calculates the needed engine power output based on the desired electric power output. The power is then divided with the actual angular velocity to get the net torque to be extracted from the single shaft. The control system inputs are the speed and the actual power generated and the outputs are fuel rate and a new value of power to be generated.

\subsection{Static verification}

The model was statically verified with data from DSA simulations. The DSA model has been carefully tuned to reflect the properties of the real microturbine and the result from DSA can therefore be regarded as highly reliable. It is also better to verify the results from the Dymola model with DSA results, since there are some variables that are not usually measured in the real microturbine. This could e.g. be the recuperator efficiency, see equation (5.5.4), where the temperature of the air after the recuperator needs to be measured. The computed values of the compressor and turbine maps have been verified by Volvo Aero Corporation AB and correction parameters have been added to the efficiency, mass flow and pressure ratio variables. Apart from that, there are additional correction parameters within Turbec $\mathrm{AB}$ on the efficiency and the mass flow of the compressor and the turbine. All these parameters have been used in the T100 model, with some modifications.

The simulations were done in the parallel mode, i.e. with the normal control system. The simulation time was set to 2000 seconds to ensure that the model had reached equilibrium. As mentioned above the tolerance was set to 1e- 6 . The 13 most important variables are used as reference variables in the verification process. The percentage error of each variable is given in the table below and is defined as:

$$
\partial x=\frac{x_{\text {model }}-x_{D S A}}{x_{D S A}} \cdot 100
$$

The absolute values are not included in this report because that information is classified.

\section{Verification of the Dymola model of the T100 microturbine at steady state}

\begin{tabular}{|c|c|c|c|c|c|}
\hline Variables & Abbrev. & units & $\begin{array}{l}\text { Perc. Err } \\
100 \mathrm{~kW}\end{array}$ & $\begin{array}{l}\text { Perc. Err } \\
70 \mathrm{~kW}\end{array}$ & $\begin{array}{l}\text { Perc. Err } \\
50 \mathrm{~kW}\end{array}$ \\
\hline rotational speed & $\mathrm{n}$ & $\mathrm{rpm}$ & $-0,238$ & 0,098 & $-0,536$ \\
\hline power generated & Pgen & $\mathrm{kW}$ & 0,000 & 0,000 & 0,000 \\
\hline fuel rate & q_released & $\mathrm{kW}$ & 0,346 & 0,914 & 0,192 \\
\hline turbine inlet temperature & TIT & $\mathrm{K}$ & $-0,852$ & $-0,876$ & $-1,031$ \\
\hline turbine outlet temperature & TOT & $\mathrm{K}$ & 0,297 & 0,080 & 0,058 \\
\hline mass flow & mdot & $\mathrm{kg} / \mathrm{s}$ & $-0,564$ & $-1,262$ & $-1,482$ \\
\hline pressure ratio compressor & $\mathrm{pr}$ & non-dim & $-0,838$ & $-1,884$ & $-3,753$ \\
\hline pressure drop & $\mathrm{dp}$ & $\mathrm{kPa}$ & 0,510 & 3,557 & 1,118 \\
\hline recuperator efficiency & eta_recup & non-dim & $-1,080$ & $-1,753$ & $-2,139$ \\
\hline gas/water hex efficiency & eta_waterhex & non-dim & $-0,775$ & 1,284 & 2,377 \\
\hline compressor efficiency & eta_comp & non-dim & 0,586 & $-0,441$ & $-0,397$ \\
\hline turbine efficiency & eta_turbine & non-dim & 0,703 & 0,524 & 0,539 \\
\hline total efficiency & eta_total & non-dim & $-0,334$ & $-0,895$ & $-0,179$ \\
\hline
\end{tabular}


Table 2: Results from static verification with data from DSA simulations

The average error is $0.61 \%, 1.2 \%$ and $1.1 \%$ for the $100 \mathrm{~kW}, 70 \mathrm{~kW}$ and $50 \mathrm{~kW}$ case. A few larger errors such as the pressure drop and the pressure ratio increase the average error significantly for the part load cases.

Power generated: This is the most important output and should therefore be exactly the same as the reference power. This is accomplished by the control system and is not really a verification of the thermodynamic model, but rather a verification of the control system.

Speed: The speed variable shows indirect efficiency of the model. If the speed of the model is lower than the speed data from DSA, the efficiency of the model is too high, because the model can have a lower speed and still produce the same electric power output.

TIT (temperature at the inlet of the turbine): This variable is used in the control system and is limiting the efficiency, since the turbine blades have a maximum temperature they are designed to sustain.

TOT (temperature at the outlet of the turbine): This variable is also used in the control system and limits the efficiency, but in this case it is the recuperator that cannot sustain too high temperatures without breaking.

Pressure ratio over the compressor: In the verification process, there has to be at least one pressure measurement. The value of the pressure ratio is better than an absolute pressure value since the pressure ratio is dimensionless and therefore not influenced by the pressure of the ambient air at the inlet. The pressure ratio over the turbine could have been used without any differences.

Added fuel power: This variable shows how much fuel has been used in the unit kW. The fuel power is preferred over the variable fuel mass flow since different fuels have different energy of combustion.

The mass flow through the compressor: This is one of the most important variables. The mass flow through the turbine could also been used without any difference.

Pressure drop from the compressor to the turbine: This variable gives information about how accurate the pressure drop models used in the heat exchangers and the combustion chamber.

Efficiency of the recuperator: This variable shows how well the recuperator works. Another variable that could have been used was total heat transferred in the recuperator, but as for a similar reason as above a dimensionless variable is preferable.

Efficiency of the gas/water heat exchanger: Same as above but for the gas/water heat exchanger instead.

Efficiency of the compressor: Since this is a variable that is difficult to model, it is important to verify the results of the model.

Efficiency of the turbine: Same as above.

Total efficiency: This important variable tells us if the complete model has the same performance as the real microturbine.

From the results of the static verification above, we see that the largest error is in the compressor mass flow and pressure ratio. In a model, where the complete microturbine system is emphasized, the errors of $2-4 \%$ can be acceptable. The errors are probably caused by errors in the ellipsoid curves that model the compressor mass flow. The pressure ratio and the mass flow for a certain speed are uniquely determined by the ellipsoid curve for that particular speed, see figure 16 . With correction parameters, the equilibrium point can be adjusted and moved along the curve. In this model, both mass flow and pressure ratio are too low, which means that the true equilibrium point lies above the model curve. Therefore the errors of the mass flow and pressure ratio have a close relation. The error of the turbine mass flow model at lower part load might also increase the error of the compressor mass flow. In Gustafsson (1998), it was also the compressor mass flow model 
that constituted the largest error, which implies that the model is not accurate enough and that the model should if possible be tuned better. The mass flow model is however already in large parts a trial and error method. The errors increase for lower speeds than the design point, $70000 \mathrm{rpm}$. This is also caused by the ellipsoid curves, where the accuracy around the design point was emphasized but also because it is much harder to model the compressor for lower speeds. From the compressor map in figure 17 we can see that small errors in pressure ratio give larger errors in mass flow, due to the horizontal shape of the ellipsoid curves, especially for lower speeds. The pressure ratio error is partly caused by errors in the pressure drop models in the combustion chamber and heat exchanger, especially at $70 \mathrm{~kW}$ load.

The value of the convection heat transfer coefficient is constant for all loads, which is not entirely true. In Gustafsson (1998), equations give that the heat transfer coefficient depends on among others the variables mass flow and dynamic viscosity, which are functions of load and temperature. The use of a constant value of the heat transfer coefficient can explain the larger error in efficiency of the recuperator for the lower loads.

All other errors are so small it does not seem worthwhile to pinpoint the cause or to come up with an explanation. Errors of static variables around $1 \%$ are very good for a dynamic model, since it is easier to get better result with just a static model, given the same amount of time to create a model.

Even though there are some errors in mass flow and pressure ratios, the most important performance variable, the total efficiency has a smaller error, because the errors cancel each other. The conclusion here will be that the model is very accurate for static purposes, but the accuracy decreases for part loads and the corresponding lower speeds. There has not been any static verification for lower part loads than $50 \mathrm{~kW}$ but the result at $30 \mathrm{~kW}$ should be approximately correct. The reason for this is that the DSA model itself has a lower degree of accuracy there, which would mean that verification with DSA data would not give meaningful results. The real microturbine has not been thoroughly tested and measured at lower part loads, since its main use is for full load or part loads down to $50 \mathrm{~kW}$. This will however change as the stand-alone mode is being implemented in future microturbines.

The model should regardless of load not be simulated under $30000 \mathrm{rpm}$ since that is the lowest data point in the turbine map. Even though the model can be simulated under $30000 \mathrm{rpm}$ the results, even though they show dynamic resemblance to a real gas turbine, should be taken with great caution.

\subsection{Dynamic verification}

The dynamic verification is more difficult than the static verification. A model is often of high order and non-linear. It is impossible to verify that a model behaves exactly as the real machine. Instead we have to limit the verification process to certain areas around equilibrium points, where the model can be linearized. When the non-linear model is linearized, we can use the powerful tools of linear system analysis to verify our model. For non-linear systems no such tools exist. The linearized system is an approximation of the non-linear system, which is very accurate only in the neighborhood of the equilibrium point.

$$
\begin{aligned}
& \dot{x}=f(x, u) \approx A x+B u \\
& y=g(x, u) \approx C x+D u
\end{aligned}
$$

where 


$$
\begin{array}{ll}
A=\left.\frac{\partial f(x, u)}{\partial x}\right|_{\left(x_{0}, u_{0}\right)} & B=\left.\frac{\partial f(x, u)}{\partial u}\right|_{\left(x_{0}, u_{0}\right)} \\
C=\left.\frac{\partial g(x, u)}{\partial x}\right|_{\left(x_{0}, u_{0}\right)} & D=\left.\frac{\partial g(x, u)}{\partial u}\right|_{\left(x_{0}, u_{0}\right)}
\end{array}
$$

where $x_{0}$ and $u_{0}$ are the values of the states and the control signals at the equilibrium point. The dynamics of a non-linear model around an equilibrium point can be therefore be approximately described by the eigenvalues of the linearized model (the eigenvalues of the A-matrix) around the same point. In order for the model to have the same dynamics as the real microturbine, the linearized model needs to have the same eigenvalues as the microturbine. Hence, similar eigenvalues give similar dynamics. The opposite is often but not always true. In Johansson (1993), we can see that two different transfer functions can give a similar impulse response.

The problem with this verification process is the difficulties to get a correct estimation of the eigenvalues through e.g. system identification of the real microturbines. Firstly, all measurement data from the microturbine are recorded under closed loop control. From Johansson (1993), we know that there are problems of non-uniqueness with identification under closed loop, even if the regulator parameters are known. For safety reasons the microturbine is usually not tested in open loop without the control system. Secondly, the data at my disposal are from experiments, which are not done with system identification in mind. The input signals are usually step responses, which normally do not fulfill the condition of persistent excitation, see Johansson (1993), but the presence of noise can help the condition to be fulfilled. Time did not permit a thorough investigation of the eigenvalues of the microturbine, so this method was not implemented.

Despite the fact that the eigenvalues do not need to be correct for the model to show a similar dynamic behavior, it is very likely that that the eigenvalues are similar if the behavior is the same. Hence, three different simulations will be used in order to verify the dynamics of the model.

The first case is a simple step response experiment. The microturbine runs at full load, $100 \mathrm{~kW}$ of electric power and is in steady state. The microturbine runs in normal parallel mode and has the corresponding control system. The power reference (plotted as dashed-dot green) is, at a given time $t$, set to $30 \mathrm{~kW}$. After another 200 seconds the reference is again set to $100 \mathrm{~kW}$. In the figures below the trajectories of the most important variables are shown. 

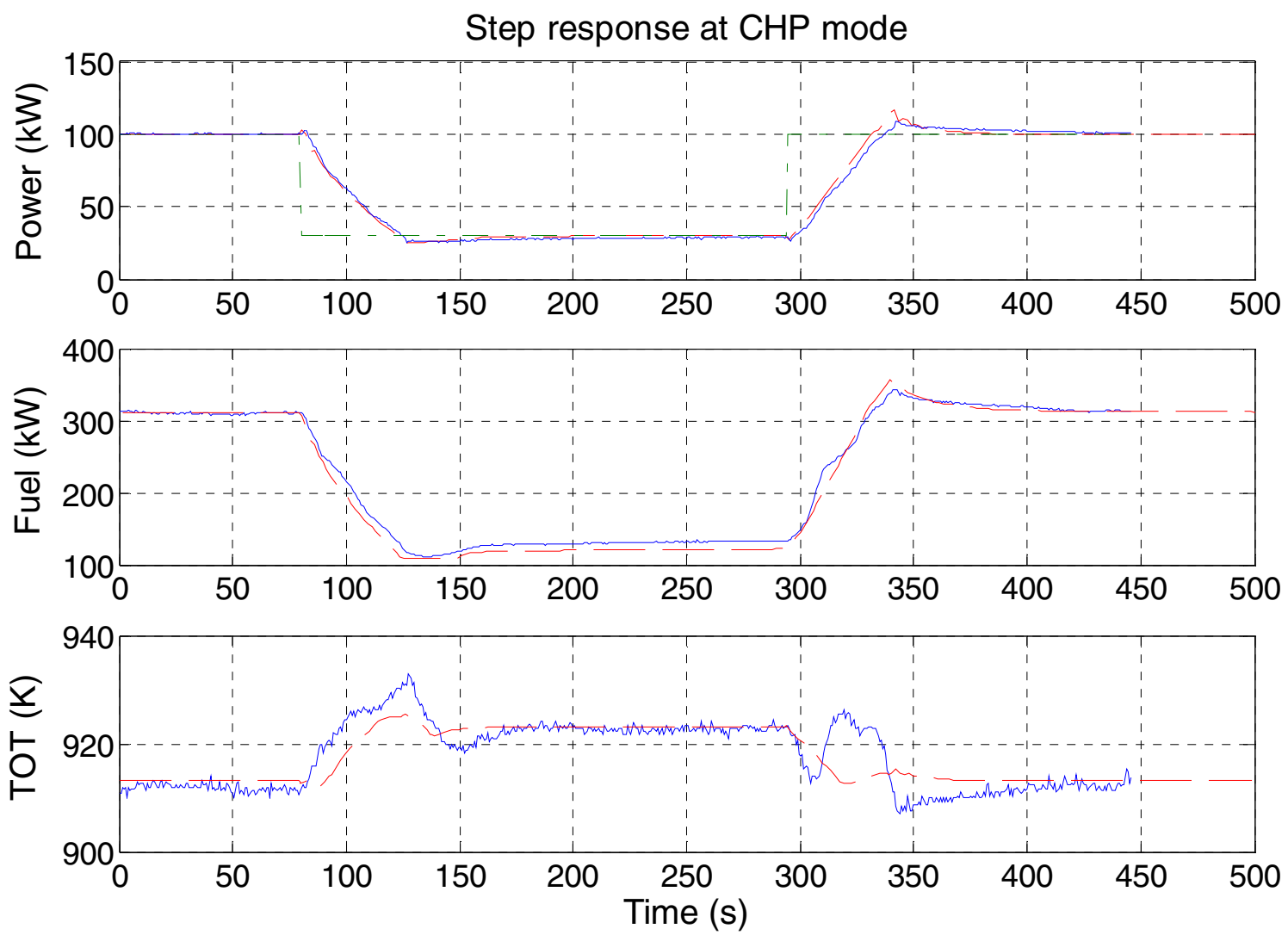

Figure 26: Step response from the model (dashed red) and the microturbine (solid blue) from a step in the power reference signal (dash-dot green)

The model shows large similarity in all variables. There is a rate limit function in the control system that prevents too large and quick power load changes and in the end limits the temperature transients the microturbine experience. The model response in TOT experiences some time delay and even a small decrease, before TOT starts to increase. This is likely due to unmodelled dynamics. At $t=320$ seconds, the measured data shows an extra bump in the TOT signal. At the same time the measured fuel value increases faster than the model's, which might cause the bump. It can also be caused by unmodelled dynamics and possibly differences in the control system model.

The second case is also a step response experiment, but now in stand-alone mode with a simpler speed regulator. The microturbine is set to $5 \mathrm{~kW}$ electric power load, i.e. some kind of idle mode. The speed is kept constant at $63000 \mathrm{rpm}$. At a given time $t$ the power reference (dashed-dot green) is set to $59.2 \mathrm{~kW}$ and is set back to $5 \mathrm{~kW}$ after another 45 seconds. 

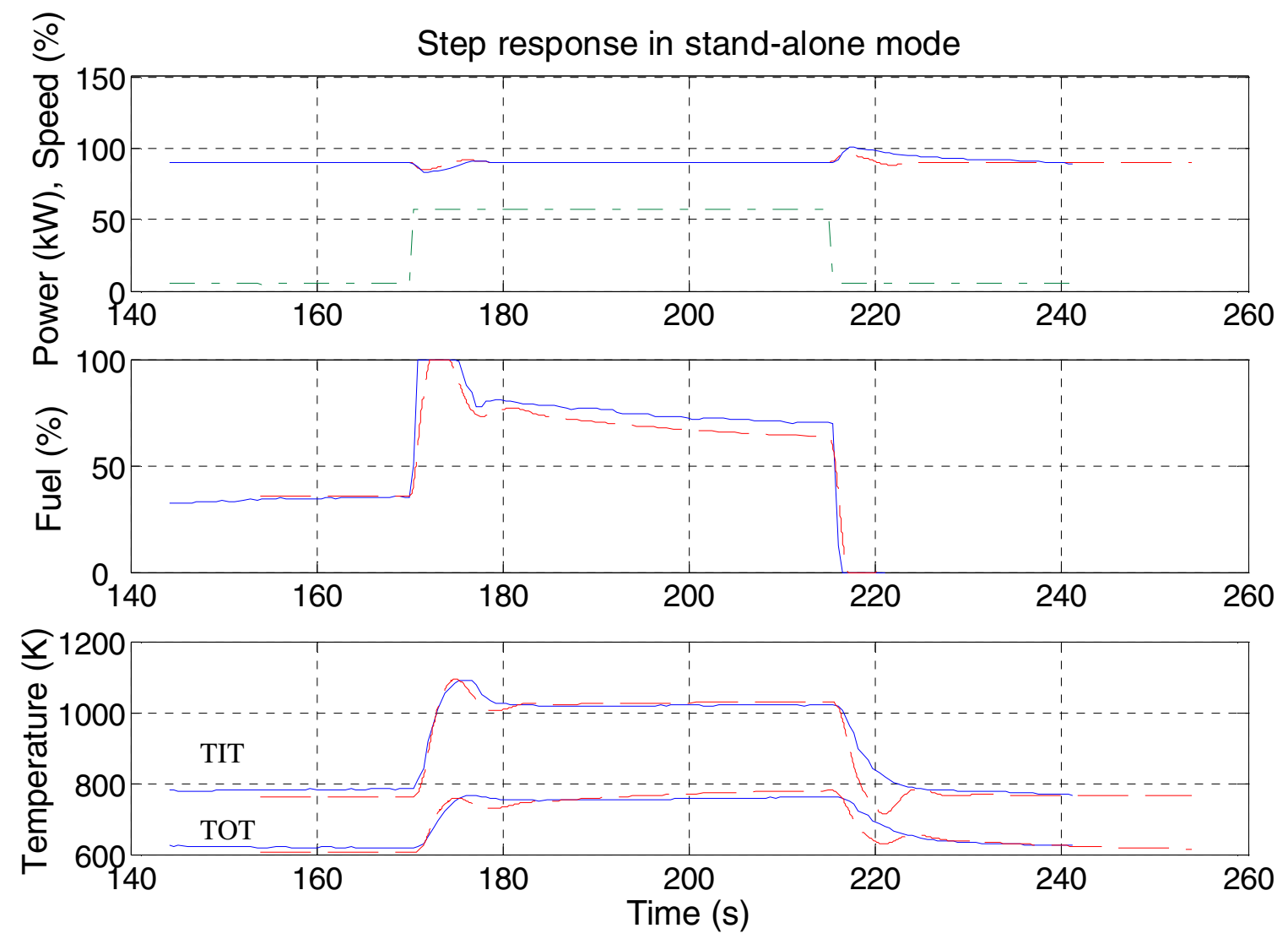

Figure 27: Step response in stand-alone mode from model (dashed red) and microturbine (solid blue) from a step (dotted green) in the power reference signal

When the load is suddenly increased, the speed drops quickly, but the regulator reacts and increases the fuel flow to the maximum value, $100 \%$. In the same way, the microturbine experiences an increase in speed when the load disappears and the fuel is cut down to a minimum, just to keep the flame alive. The difference between the model and the microturbine in speed around 220 seconds depends probably on differences in the control system settings. The difference in dynamics between the TIT signal and the TOT signal is very interesting. In the original turbine model, the turbine is just an algebraic relation between speed, mass flow and pressure. Then the TIT and TOT signals would have exactly the same dynamics. As we can see from experimental data from the microturbine (solid blue), there is additional dynamics similar to a first order filter. The reason behind this is the extra heat storage in the turbine rotor and the turbine diffuser, see section 3.2. This is modelled by an extra heat exchanger. There is an interesting difference between the model and the measured data in TOT and TIT, right after the step at 220 seconds. The measured temperatures decrease slower, which could indicate that the extra heat storage effect described above is larger than in the model or that the time constant of the temperature sensors are larger.

The third and last case is brake test verification. The microturbine is run at part load. At a given time $t$ the electric power is disconnected to simulate a power circuit failure. When the power is disconnected, the fuel valves are closed immediately. To prevent the microturbine from over speed, i.e. speeds over $70000 \mathrm{rpm}$, a brake chopper is switched on. The brake chopper consists of resistors, which brake and dissipate the kinetic energy of the machine via the generator. The brake chopper is therefore modelled exactly as the generator, i.e. a power reference that gives the 
engine power output. With this simulation, the most important dynamic is the speed at which the microturbine decelerates. The axes of the figure below are removed for confidentiality.

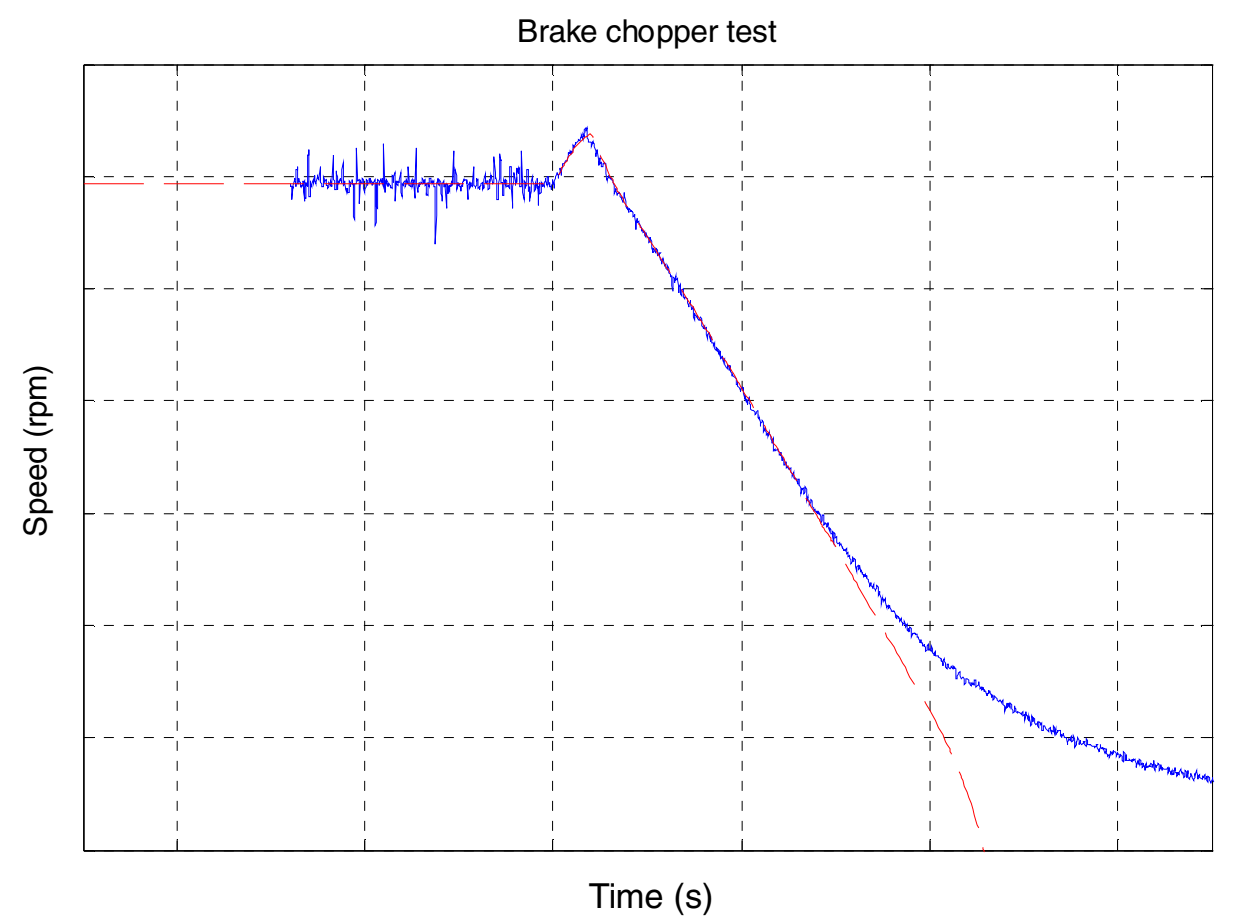

Figure 28: Brake chopper test with data from the model (dashed red) and the microturbine (solid blue) when the power grid is disconnected at time $t=600$ seconds.

The model value of the speed shows great similarity with the measured values down to 35000 rpm, which is the within the valid range of the model. The difference can be explained by two separate causes. Firstly, there are larger model errors at lower speeds, especially since data in the compressor and turbine map are given down to 21000 and $30000 \mathrm{rpm}$ respectively. Secondly and possibly the largest error is a difference in the simple brake chopper model (the power it dissipates) and the control system model. As described in section 5.10 and figure 25 the desired engine power output is divided with the angular velocity to get the brake chopper torque. When the speed is decreasing faster than the desired power output, the torque is increased even more. As the speed goes to zero, the torque goes in theory to infinity. This effect can be seen in figure 28 , where the speed drops very quickly in the end.

\section{Conclusions and Summary}

In this chapter I will discuss the results given in the previous chapter and give a summary of the models used in this thesis.

The mass flow of the compressor is modelled with fitted curves, which parameters can be evaluated by polynomial functions, see figure 17 . The efficiency of the compressor is modelled as parabolic degradation curves, which can be seen in figure 18. The mass flow through the turbine is modelled as choked (at a maximum) through the entire working range, see equation (5.4.1) and figure 20. The efficiency of the turbine is computed through interpolation, see equation (5.4.4). The heat exchangers in the microturbine are modelled as pipes with heat convection and conduction in one dimension, see figure 22. The increase in enthalpy of the fluid in the combustion chamber is based on reaction formulas and the corresponding energy released during 
combustion, see equation (5.8.10). The generator is modelled through a torque that is put on the shaft of the compressor and the turbine, see equation (5.10.2).

\subsection{Static verification}

The values of the model are very close to the data from DSA. The average errors of the variables at the different loads are $0.6 \%, 1.2 \%$ and $1.1 \%$ for the $100 \mathrm{~kW}, 70 \mathrm{~kW}$ and $50 \mathrm{~kW}$ case respectively. The emphasis in this thesis has been on the complete model of the microturbine over accurate submodels of individual components, in order to get a complete model running early in the project.

\subsection{Dynamic verification}

The model shows a great similarity with the measured values from the real microturbine. The task of tuning a dynamic model is an incredible difficult task, since there are a vast number of parameters with strong coupling effects to each other. As discussed earlier, the verification should be done in open loop simulations, so that differences in the control system models would not affect the results. I have chosen the verification simulations, so that as much as possible of the dynamics of the model should be verified. Still there are more verification simulations to be done if one wants to cover all possible operations and if the dynamics should be verified beyond reasonable doubt, then system identification must be made on the microturbine.

\subsection{Valid operating ranges for the model}

One of the most important things to know about a model is not exactly how accurate it is, but in which areas it is verified and validated. All the components have a range of operation and the limiting components are listed below.

The medium used is a mixture of carbon dioxide, water vapor, oxygen and nitrogen. The data that gives the thermodynamic property of a substance is valid at temperatures from $200 \mathrm{~K}$ up to 6000 $\mathrm{K}$. The water vapor model does not handle the condensation of water in the fog region for temperatures below the dew point temperature, but the compressor quickly heats the gas so the possible effect can be neglected.

The compressor and turbine data implemented in the model cover speeds from $74000 \mathrm{rpm}$ down to $21000 \mathrm{rpm}$ and $30000 \mathrm{rpm}$ respectively. The model should therefore not be used under 30000 $\mathrm{rpm}$, even though it is numerically possible to simulate the microturbine down to $15000 \mathrm{rpm}$. Between $30000 \mathrm{rpm}$ and $15000 \mathrm{rpm}$ the model have a behavior that resembles a gas turbine, but the results should used with great caution.

The model is statically verified down to $50 \mathrm{~kW}$ part load with high accuracy. It is very likely that the model has an acceptable accuracy even down to $30 \mathrm{~kW}$ part load.

\section{Possible Applications and Future Work}

A model is never perfect. There are always things to be improved, but there is also a trade-off between time spent on a component and the component's functionality and value in the model. The same reason goes for more detailed modelling. A functional model as been developed and the model can surely be made more detailed, but the question is, is it worth the extra time spent? If it is, then one might do it later when the need arises. But the most important thing is to get a complete model running and then choose what part we want to study further. 
As described earlier in the thesis, there are many possibilities for this model. After verification and testing of the model, there are a lot of possible applications:

- The model can be used in What-if studies at internal and external dynamic changes, i.e. what happens e.g. with the temperature after the turbine if the load changes or if the temperature of the ambient air is suddenly changed

- The model can be used to design control strategies, e.g. when using a brake chopper.

- The model can be used to evaluate new developments in the control system for the standalone mode, before tried on a real microturbine.

Suggestions for future work:

- The existing rate limit model does work for discrete time, but in continuous time it can only be used if the source is analytically differentiable, e.g. a sinusoidal or ramp signal. The model should be able to deal with signals that are not directly differentiable, i.e. signals from time tables or if the rate limit model is placed far away from the source.

- Developing an operator interface with the LabVIEW program in real time for operator training.

- With the LabVIEW application, it can be possible to initiate hardware/software failures, so that service technicians/engineers can learn how the gas turbine behaves with a similar failure.

- The bypass function was never fully modelled and should be completed if such interest arises.

- The medium models can be changed into models that uses fewer individual substances and instead a constant composition, thus reducing the number of states in the model. With fewer states the simulation time will decrease and the model will be easier to linearize and to use in state-space equations in control design.

- The heat exchanger might be modelled with the log-mean temperature method. It might then be possible to reduce the number of discretisations used in the heat exchanger and therefore the number of states in the model. It is however not obvious that the dynamic behavior will not be changed.

- Development of a model of the power electronics so that the complete machine can be simulated against a power grid.

- With a power electronics model, the complete power plant can be used to design and verify control strategies for power production with several machines connected in parallel.

- The model can be linearized and then used in a TOT estimator in the flame-out detection system.

- The model can be linearized and put in a state-space form and then used in theoretical control design

\section{References}

1. Cohen H, Rogers GFC and Saravanamuttoo HIH: "Gas Turbine Theory". Longman Group Ltd. $4^{\text {th }}$ ed. London, 1996

2. Cengel Y.A and Boles M.A: "Thermodynamics, An Engineering Approach". WCB/McGrawHill $3^{\text {rd }}$ ed. New York, 1998

3. DeWitt D.P and Incropera F.P: "Fundamentals of Heat and Mass Transfer". Wiley $4^{\text {th }}$ ed. New York, 1996

4. Dynasim AB: "Dymola, Dynamic Modeling Laboratory - User's Manual". Dynasim AB Lund, 2001 
5. Eborn J: "On Model Libraries for Thermo-hydraulic Applications". Doctoral Thesis, Lund University ISRN LUTFD2 / TFRT-1061-SE Lund, 2001

6. Eborn J, Tummescheit H and Åström K.J: "Physical System Modeling with Modelica ${ }^{\mathrm{TM}}$ ", Lund, http://www.control.lth.se/ hubertus/ThermoFluid, 1999

7. Eborn J, Tummescheit $\mathrm{H}$ and Wagner F: "ThermoFlow, A Thermo-Hydraulic Library in Modelica, Documentation" http://www.control.lth.se/ hubertus/ThermoFluid, 2000

8. Fox R.W and McDonald A.T: "Introduction to Fluid Mechanics". Wiley $5^{\text {th }}$ ed. New York, 1998

9. Gordon S, and McBride B.J: "Computer program for calculation of complex chemical equilibrium compositions and applications", NASA Technical Reports Report Number: NASA-RP-1311 E-8017 NAS 1.61:1311, NASA Lewis Research Center, 1994

10. Gustafsson J.O: "Static and Dynamic Modelling of Gas Turbine in Advanced Cycles". Licentiate Thesis, Lund University ISRN LUTMDN/TMVK 98/7030-SE Lund, 1998

11. Johansson R: "System Modelling \& Identification". Prentice Hall $1^{\text {st }}$ ed. New Jersey, 1993

12. Malmquist A: "Analysis of a Gas Turbine Driven Hybrid Drive System for Heavy Vehicles". Doctoral thesis, The Royal Institute of Technology ISSN-1102-0172, Stockholm, 1999

13. Modelica Association: "Modelica TM - A Unified Object-Oriented Language for Physical Systems Modeling, Language Specification and Tutorial" www.modelica.org, 2000

14. Pérez Gómez A.A: "Modelling of a Gas Turbine with Modelica ${ }^{\mathrm{TM}}$ ". Master Thesis, Lund University ISRN LUTFD2/TFRT—5668—SE, Lund, 2001

15. Thomas P: "Simulation of Industrial Processes for Control Engineers". ButterworthHeinemann $1^{\text {st }}$ ed. Oxford, 1999

16. Tummescheit H: "Object Oriented Modeling - Design and Implementation of Libraries using Modelica". Doctoral thesis, to be published, Lund University, 2002

17. Wagner F: "The Free Modelica Editor". http://www.et.dtu.dk/fme, 2000

18. Wagner W and Kruse A: "Properties of Water and Steam", IAPWS-IF97. Springer-Verlag, Berlin, 1998 
